\title{
$\beta$-hydroxybutyrate supports synaptic vesicle cycling but reduces endocytosis and exocytosis in rat brain synaptosomes
}

Sviatlana V. Hrynevich ${ }^{*}$, Tatyana V. Waseem*, Audrey Hébert", Luc Pellerin", Sergei V. Fedorovich*

*Laboratory of Biophysics and Engineering of Cell, Institute of Biophysics and Cell Engineering, Akademicheskaya St., 27, Minsk 220072, BELARUS

\# Department of Physiology, University of Lausanne, Lausanne, Switzerland

Corresponding author: Dr. Sergei V. Fedorovich, Institute of Biophysics and Cell Engineering, Akademicheskaya St., 27, Minsk 220072, BELARUS

Tel: + 375-172-84-2252; Fax: + 375-172-84-2359

Email: fedorovich@ibp.org.by 


\begin{abstract}
The ketogenic diet is used as a prophylactic treatment for different types of brain diseases, such as epilepsy or Alzheimer's disease. In such a diet, carbohydrates are replaced by fats in everyday food, resulting in an elevation of blood-borne ketone bodies levels. Despite clinical applications of this treatment, the molecular mechanisms by which the ketogenic diet exerts its beneficial effects are still uncertain. In this study, we investigated the effect of replacing glucose by the ketone body $\beta$-hydroxybutyrate as the main energy substrate on synaptic vesicle recycling in rat brain synaptosomes. First, we observed that exposing presynaptic terminals to nonglycolytic energy substrates instead of glucose did not alter the plasma membrane potential. Next, we found that synaptosomes were able to maintain the synaptic vesicle cycle monitored with the fluorescent dye acridine orange when glucose was replaced by $\beta$-hydroxybutyrate. However, in presence of $\beta$-hydroxybutyrate, synaptic vesicle recycling was modified with reduced endocytosis. Replacing glucose by pyruvate also led to a reduced endocytosis. Addition of $\beta$-hydroxybutyrate to glucose-containing incubation medium was without effect. Reduced endocytosis in presence of $\beta$-hydroxybutyrate as sole energy substrate was confirmed using the fluorescent dye FM2-10. Also we found that replacement of glucose by ketone bodies leads to inihibition of exocytosis, monitored by FM2-10. However this reduction was smaller than the effect on endocytosis under the same conditions. Using both acridine orange in synaptosomes and the genetically encoded sensor synaptopHluorin in cortical neurons, we observed that replacing glucose by $\beta$ hydroxybutyrate did not modify the $\mathrm{pH}$ gradient of synaptic vesicles. In conclusion, the nonglycolytic energy substrates $\beta$-hydroxybutyrate and pyruvate are able to support synaptic vesicle recycling. However, they both reduce endocytosis. Reduction of both endocytosis and exocytosis together with misbalance between endocytosis and exocytosis could be involved in the anticonvulsant activity of the ketogenic diet.
\end{abstract}

Keywords: synaptosomes, ketogenic diet, ketone bodies, endocytosis, synaptic vesicles, $\beta$-hydroxybutyrate 


\section{LIST OF ABBREVIATIONS}

APV - 2-amino-5-phosphonovaleric acid

BHB - $\beta$-hydroxybutyrate

CNQX - 6-cyano-7-nitroquinoxaline-2,3-dione

DiSC3(5) - 3,3'-dipropylthiadicarbocyanine

FCCP - carbonyl cyanide 4-(trifluoromethoxy)phenylhydrazone

GW - glucose withdrawal

MCT - monocarboxylate transporter

Pyr - pyruvate

R.U. - relative units

Tris - Tris(hydroxymethyl)aminomethane 


\section{INTRODUCTION}

Brain is an expensive organ from a bioenergetic point of view. In humans, the brain represents only $2 \%$ of the body mass, yet it uses about $20-25 \%$ of the oxygen and glucose consumed at rest (Kety and Schmidt, 1946; Attwell and Laughlin, 2001; Harris et al., 2012). Glucose is the main energy substrate for the brain, but lactate, pyruvate, and the ketone bodies can also be used in certain situations (Elliott et al., 1937; Rollestone and Newsholme, 1967; Hawkins et al., 1971; Izumi et al., 1998; Castro et al., 2009; Pellerin and Magistretti, 2012). Ketone bodies are generally consumed in the early stages of development; however, sometimes they can contribute to satisfy energy demands in adults, e.g. during starvation (Hawkins et al., 1971; Izumi et al., 1998; White and Venkatesh, 2011).

The ketogenic diet was developed as a putative prophylactic treatment for various brain diseases, from epilepsy to Alzheimer's disease (Peterman, 1924; White and Venkatesh, 2011; McNally and Hartman, 2012; Stafstrom and Rho, 2012; Gano et al., 2014). In the ketogenic diet, carbohydrates are replaced by fats in daily meals, leading to an increase of ketone body synthesis by the liver followed by a rise in their blood-borne concentration. Circulating ketone bodies are mainly represented by $\beta$-hydroxybutyrate and acetoacetate. Although beneficial in several cases, compliance to the ketogenic diet can be complicated for patients, and it causes some adverse effects in the liver and kidney (Ballaban-Gil et al., 1998; Sampath et al., 2007; Garbow et al., 2011; White and Venkatesh, 2011). Currently, it is impossible to replace it by selective drugs because the molecular and cellular mechanisms of this treatment have not been determined precisely (White and Venkatesh, 2011; McNally and Hartman, 2012; Stafstrom and Rho, 2012; Gano et al., 2014). Ketone bodies are believed to be more effective energy substrates than glucose under certain circumstances and are able to improve neuronal bioenergetics (Holmgren et al., 2010; Gano et al., 2014). However, alternative hypotheses proposed to explain these effects involve either modification of synaptic vesicle loading (Juge et al., 2010) or regulation by mitochondrial apoptotic proteins such as BCL2-associated agonist of cell death (Gimenez-Cassina et al., 2012).

Neurons communicate with each other through neurotransmitter release. Neurotransmitters are stored in synaptic vesicles, which are exocytosed in response to a rise in cytosolic calcium levels (Sudhof, 2004, 2013). Synaptic neurotransmission is an energydependent process (Attwell and Gibb, 2005; Harris et al., 2012). In addition to ion transport which is the main consumer of ATP, different stages of the synaptic vesicle cycle, for 
instance, synaptic vesicle loading with neurotransmitters, priming, and endocytosis, are also energy-consuming processes (Sudhof, 2004, 2013; Attwell and Gibb, 2005; Harris et al., 2012). Ketone bodies are able to support the maintenance of plasma membrane potential in neurons and synaptic activity in brain slices (Izumi et al., 1998; Holmgren et al., 2010). Furthermore, it was shown that they can prevent synaptic dysfunction in case of mitochondrial impairments (Kim et al., 2010). Pyruvate, an alternative nonglycolytic energy substrate, is able to support synaptic vesicle recycling in neurons and synaptosomes (Morgenthaler et al., 2006; Tarasenko et al., 2006). However, concerning nonglycolytic energy substrate, two questions are still not resolved. First, are ketone bodies able to support synaptic vesicle recycling and synaptic vesicle maintenance ? And second, do nonglycolytic energy substrates fuel synaptic vesicle cycle similarly to glucose?

In the present paper, we investigated the influence of DL- $\beta$-hydroxybutyrate on synaptic vesicle recycling in rat brain synaptosomes revealed with the fluorescent dye acridine orange (Zoccarato et al., 1999) or FM2-10 (Cousin and Robinson, 2000). We used isolated neuronal presynaptic endings termed synaptosomes as many properties of intact terminals are preserved including synaptic vesicle recycling or calcium-dependent neurotransmitter release, while ion gradients are similar (Waseem et al., 2005, 2007; Wilhelm et al., 2014). Direct evidences that synaptosomes are able to utilize ketone bodies are not currently available. However, it was shown that synaptosomes exhibit monocarboxylate transporters (MCTs) activities (McKenna et al., 1998) some of which might enable them to utilize ketone bodies as energy substrates (Pierre and Pellerin, 2005; Bergersen, 2015).

\section{MATERIALS AND METHODS.}

\subsection{Materials}

DL- $\beta$-hydroxybutyric acid sodium salt, pyruvate, 6-aminonicotinamide, 3,3' dipropylthiadicarbocyanine (DiSC3(5)), carbonyl cyanide 4-(trifluoromethoxy) phenylhydrazone (FCCP), bafilomycin A1, 2-amino-5-phosphonovaleric acid (APV), 6cyano-7-nitroquinoxaline-2,3-dione (CNQX) were purchased from Sigma (St.Louis, MO, USA). 4-(2-Hydroxyethyl)piperazine-N'-1-ethanesulfonic acid (HEPES) was obtained from Merck (Darmstadt, Germany). Acridine orange was obtained from Serva (Heidelberg, Germany). FM2-10 was obtained from Invitrogene (Carlsbad, CA, USA). 
Tris(hydroxymethyl)aminomethane (Tris) was obtained from BDH (Poole, UK). Plasmid of super-ecliptic, non-ratiometric synaptopHluorin was kindly provided by Prof. Gero Miesenbock (University of Oxford, Oxford, UK).

\subsection{Preparation of synaptosomes}

Synaptosomes were isolated from brain hemispheres of 12-16-week-old male Wistar rats according to Hajos (1975). Stock suspensions of synaptosomes $(10 \mathrm{mg} / \mathrm{mL})$ were suspended in different synaptosomal media (see Table I) and kept on ice. Synaptosomes were suspended in glucose-containing, ketone-body containing or pyruvate-containing incubation mediums (Table I) in corresponding experiments. Prior to each experiment, synaptosomes were preincubated for $10 \mathrm{~min}$ at $37^{\circ} \mathrm{C}$ and then kept on ice and used within 3 hours.

Animal experiments were carried out in accordance with EU Directive 2010/63/EU. Institutional Animal Ethics Committee at Institute of Biophysics and Cell Engineering NASB approved all animal experiments

\subsection{Preparation of primary cultures of mouse cortical neurons}

Primary cultures of mouse cortical neurons were prepared according to the modified protocol of Brewer et al. (1993) and Brewer (1995) with some modifications (Robinet and Pellerin, 2010). Briefly, after decapitation and brain dissection, cortices were mechanically dissociated in phosphate-buffered saline supplemented with glucose (composition in mM: 150 glucose, $3 \mathrm{KCl}, 1.5 \mathrm{KH}_{2} \mathrm{PO}_{4}, 7.9 \mathrm{Na}_{2} \mathrm{HPO}_{4}, 33$ glucose, $100 \mathrm{U} / \mathrm{ml}$ penicillin and $0.1 \mathrm{mg} / \mathrm{ml}$ streptomycin). Cells were plated onto poly-ornithine-coated coverslips to produce cultures highly enriched in neurons and cultured in neurobasal-B27 medium supplemented with 0.5 mmol/L glutamine (Brewer et al., 2003).

\subsection{Investigation of plasma membrane potential}

Putative effects on the plasma membrane potential were investigated using the fluorescent dye DiSC3(5) according to Waseem and Fedorovich (2010) with some modifications (Hrynevich et al., 2015). An aliquot of the synaptosomal suspension (200 $\mu$ l) was added to the cuvette containing $2 \mathrm{~mL}$ of the corresponding incubation medium (see Table I) with $2 \mathrm{mM}$ $\mathrm{CaCl}_{2}$. After $1 \mathrm{~min}, 1 \mu \mathrm{M}$ of DiSC3(5) was added to the cuvette. Fluorescence intensity was 
recorded in kinetics mode at $\lambda_{\mathrm{ex} / \mathrm{em}}=640 / 688 \mathrm{~nm}$ using a spectrofluorimeter Cary Eclipse ("Varian", USA) under constant stirring and at $37^{\circ} \mathrm{C} . \mathrm{KCl}$ was added to the cuvette 1 minute after addition of the dye. The same quantity of $\mathrm{NaCl}$ was added during control experiments. The control curve was extracted from the experimental curve. Measurements were repeated at least 8 times for each investigated energy substrate with similar results.

\subsection{Investigation of synaptic vesicle recycling and synaptic vesicle $\mathrm{pH}$ gradient}

Synaptic vesicle recycling was detected using the fluorescent dye acridine orange according to Zoccarato et al. (1999) with some modifications (Waseem et al., 2005). Fluorescence was recorded in kinetics mode using a spectrofluorimeter Cary Eclipse ("Varian", USA) at $37^{\circ} \mathrm{C}$ under constant stirring. Recording parameters were as follows: $\lambda_{\mathrm{ex}}-$

$494 \mathrm{~nm}, \lambda_{\mathrm{em}}-529 \mathrm{~nm}$. $200 \mu \mathrm{l}$ of synaptosomal suspension were added to $1800 \mu \mathrm{l}$ of corresponding incubation medium (see Table I) containing $2 \mathrm{mM} \mathrm{CaCl}_{2}$ to obtain the final protein concentration of $1 \mathrm{mg} / \mathrm{mL}$. Next, $20 \mu \mathrm{l}$ of aqueous solution of acridine orange were added to obtain the final concentration of $5 \mu \mathrm{M}$. The suspension was incubated for 10 minutes to allow equilibration between synaptosomes and dye. Thereafter, various additions were made. Sixty $\mathrm{mM} \mathrm{KCl}$ was used for stimulation of exocytosis and compensatory endocytosis. One hundred nM Bafilomycin A1 was used for investigation of synaptic vesicle $\mathrm{pH}$ gradient. The same quantity of vehicle or appropriate amount of $\mathrm{NaCl}$ was added during control experiments. The control curve was extracted from the experimental curve. Measurements were repeated at least 7 times for each investigated energy substrate with similar results.

\subsection{Measurement of endocytosis and exocytosis with the fluorescent dye FM2-10.}

Endocytosis was measured according to Cousin and Robinson (2000) using the fluorescent dye FM2-10 with some modifications (Waseem et al., 2005). The synaptosomal pellet was resuspended in the corresponding depolarizing medium (see Table I) to a final concentration of 5-8 mg protein $/ \mathrm{ml}$. Synaptosomes were loaded with $200 \mu \mathrm{M} \mathrm{FM} 2-10$ for $10 \mathrm{~min}$ at $30^{\circ} \mathrm{C}$. For initiation of endocytosis, $3 \mathrm{mM} \mathrm{CaCl}_{2}$ was added and the suspension was incubated for an additional $3 \mathrm{~min}$. Excess of extracellular dye was removed by sedimentation in isotonic medium A (10 min, 12,000 g). This process was repeated three times. The synaptosomal pellet was resuspended in medium A to a final concentration of $0.125 \mathrm{mg}$ protein $/ \mathrm{ml}$ and was placed in darkened glass to prevent photobleaching. In order to monitor dye release, $200 \mu$ of 
FM2-10 loaded synaptosomes were added to $1800 \mathrm{ml}$ glucose-containing medium A (see Table I) containing $2 \mathrm{mM} \mathrm{CaCl}_{2}$ or $2 \mathrm{mM}$ EGTA. Subsequent treatments were added directly to a cuvette. Fluorescence intensity was recorded in kinetics mode at $\lambda_{\mathrm{ex} / \mathrm{em}}=472 / 563 \mathrm{~nm}$ and spectral width of slits ex/em $=5 / 10 \mathrm{~nm}$ with a spectrofluorimeter Cary Eclipse ("Varian', USA) at $37^{\circ} \mathrm{C}$ under constant stirring. Exocytosis was stimulated by adding $60 \mathrm{mM} \mathrm{KCl}$. The appropriate amount of $\mathrm{NaCl}$ was added in control experiments. The control curve was extracted from the experimental curve. The influence of $\beta$-hydroxybutyrate on exocytosis was measured by a similar protocol (Cousin and Robinson, 2000; Waseem et al., 2005) except for the dye that was loaded in the control glucose-containing medium and released in the glucoseor $\beta$-hydroxybutyrate-containing medium. Measurements were repeated at least 5 times for each investigated energy substrate with similar results.

\subsection{SynaptopHluorin imaging in cultured cortical neurons}

$\mathrm{pH}$ gradient of synaptic vesicles in cultured cortical neurons was estimated using the genetically encoded sensor synaptopHluorin (Miesenbock et al., 1998; Burron, 2006). Material for neuronal transfection (Maxi prep) was obtained by using the Macherey Nagel kit according to manufacturer's instructions. Neurons were transfected on day 7 in vitro using 'Lipofectamine ${ }^{\circledR}$, (Life Technologies) according to manufacturer's instructions for a period of 12 hours before being used for imaging experiments. Neurobasal medium was replaced by the corresponding neuronal imaging solutions (see Table I) one hour prior to starting the experiment. Neurons grown on coverslips were mounted in a closed perfusion chamber for imaging experiments. Experiments were performed at room temperature on the stage of an inverted microscope (Axiovert 100M, Carl Zeiss) with a 63 X 1.3 NA Plan Neofluar objective (Carl Zeiss). Fluorescence was excited and observed through installed FITS filter cube (excitation 450-490 nm, emission $515 \mathrm{~nm}$ ) using Hammamatsu Orca ER cooled CCD camera. ROIs were taken manually as synaptic boutons responding to FCCP with fluorescence increase. Measurements were repeated at least 8 times for each investigated energy substrate with similar results.

\subsection{Other methods}

Protein concentration was assayed according to Lowry et al. (1951) using bovine serum albumin as a standard. Data are presented as mean \pm S.E.M. where indicated, statistical 
significance was evaluated by one-way analysis of variance (ANOVA) followed by Tukey post-hoc test. SynaptopHluorin images were analyzed with the 'Image J' software.

\section{RESULTS}

\subsection{Effect of glucose replacement by nonglycolytic energy substrates on synaptosomal plasma} membrane potential

It was observed that the replacement of glucose by $\beta$-hydroxybutyrate as the sole energy substrate does not lead to a significant change in the plasma membrane potential of synaptosomes, as revealed with the voltage-sensitive fluorescent dye DiSC3(5). However, replacement of glucose by pyruvate significantly decreases the accumulation of the dye (Fig. $1)$.

3.2 Effect of glucose replacement by nonglycolytic energy substrates on synaptic vesicle recycling in synaptosomes

The fluorescent dye acridine orange was used to monitor the synaptic vesicle cycle. As shown in Figure 2a, a potassium-induced depolarization evokes a fluorescence increase reflecting exocytosis (Zoccarato et al., 1999; Waseem et al., 2005), followed by fluorescence decline reflecting compensatory endocytosis (Zoccarato et al., 1999; Waseem et al., 2005). Replacement of glucose by $\beta$-hydroxybutyrate leads to a small, but statistically significant, stimulation of exocytosis (Fig. 2a, b). Under the same conditions, endocytosis was reduced (Fig. 2a, c). Incubation of synaptosomes in presence of both $\beta$-hydroxybutyrate and glucose does not significantly change both exocytosis and endocytosis although exocytosis had a tendency to be enhanced like with $\beta$-hydroxybutyrate alone (Fig. 2b, c). Replacement of glucose by the other nonglycolytic energy substrate pyruvate only led to reduced endocytosis (Fig. 2c). Incubation of synaptosomes in glucose-free incubation medium containing either $\beta$ hydroxybutyrate or pyruvate resulted in a statistically significant decrease of the endocytosis/exocytosis ratio (Fig. 2d). 
3.3 Effect of glucose replacement by $\beta$-hydroxybutyrate on endocytosis and exocytosis in synaptosomes monitored with the fluorescent dye FM2-10

Inhibition of endocytosis in $\beta$-hydroxybutyrate-fueled synaptosomes was further confirmed using the fluorescent dye FM2-10. The dye was loaded into synaptosomes by potassium-induced depolarization in control incubation medium and in medium with glucose replaced by $8 \mathrm{mM} \beta$-hydroxybutyrate. After washing, dye-loaded synaptosomes were transferred to a standard glucose-containing incubation medium, and the dye was released by a second potassium-induced depolarization. Background level of fluorescence was similar when synaptosomes were loaded in $\beta$-hydroxybutyrate-containing medium (data not shown). Replacing glucose by $\beta$-hydroxybutyrate as energy substrate significantly reduced synaptic vesicles FM2-10 uptake in synaptic vesicles by two thirds, indicating a reduction of endocytosis (Fig 3a, b). This effect was even more pronounced than the reduction of endocytosis observed using the fluorescent dye acridine orange (Fig. 2c). We found that release of the dye in $\beta$-hydroxybutyrate-containing incubation medium was also inhibited by about $50 \%$ (Fig. 3c). In the case of exocytosis experiments, the dye was loaded into synaptosomes by potassium-induced depolarization in control incubation medium. After washing, dye-loaded synaptosomes were transferred to a standard glucose-containing incubation medium or in $\beta$-hydroxybutyrate containing medium, and the dye was released by a second potassium-induced depolarization.

3.4 Effect of glucose replacement by $\beta$-hydroxybutyrate on synaptic vesicle $\mathrm{pH}$ gradient in synaptosomes

Addition of $100 \mathrm{nM}$ of bafilomycin A1, an inhibitor of vesicular ATPase, leads to a significant increase of acridine orange fluorescence, reflecting dissipation of the synaptic vesicle $\mathrm{pH}$ gradient (Fig. 4a). Replacement of glucose by $\beta$-hydroxybutyrate gave rise to an almost identical response (no significant difference), indicating that it can sustain the formation of a similar synaptic vesicle $\mathrm{pH}$ gradient as glucose (Fig. 4b).

3.5 Estimation of synaptic vesicle pH gradient in cortical neurons using the genetically encoded sensor synaptopHluorin

In order to confirm the aforementioned results obtained with synaptosomes, it was examined whether $\beta$-hydroxybutyrate provided as sole energy substrate could support 
synaptic vesicle $\mathrm{pH}$ gradient in situ in presynaptic endings of cortical neurons. Application of the proton ionophore FCCP $(10 \mu \mathrm{M})$ led to a fluorescence increase in synaptic boutons, indicating a dissipation of synaptic vesicle $\mathrm{pH}$ gradient (Figs. 5a-c). We found 22 responding synaptic boutons in the case of control glucose-containing medium, 41 responding synaptic boutons in the case of $\beta$-hydroxybutyrate-containing medium, and 8 responding synaptic boutons in the case of energetic substrate-free incubation medium. Exposing neurons to an energy substrate-free incubation medium for one hour led to the almost complete disappearance of the FCCP response, sign that the $\mathrm{pH}$ gradient could not be formed properly in the absence of an energy source (Fig. 5d). Incubation in presence of $\beta$-hydroxybutyrate completely rescued the fluorescence increase response normally obtained with glucose (Fig. $5 \mathrm{~d})$.

\section{DISCUSSION}

$\beta$-Hydroxybutyrate accounts for $70 \%$ of the different ketone bodies synthesized by the liver (Dedkova and Blatter, 2014). Therefore, this compound can be considered as the main ketone body made available and used by the brain in prolonged fasting conditions as well as under a ketogenic diet. Resting level of $\beta$-hydroxybutyrate in blood is about $0.1 \mathrm{mM}$. Several hours of starvation can increase it up to $1 \mathrm{mM}$. Long starvation or exposure to a ketogenic diet leads to circulating ketone bodies blood values up to 5-8 $\mathrm{mM}$ (White and Venkatesh, 2011; Dedkova and Blatter, 2014; Paoli et al., 2014). It was shown previously that incubation in presence of $4 \mathrm{mM}$ of the alternative nonglycolytic energy substrate pyruvate instead of glucose is able to support the main functional characteristics of synaptosomes (Tarasenko et al., 2006). Therefore, it was decided to use $4 \mathrm{mM}$ pyruvate and $8 \mathrm{mM}$ DL- $\beta$-hydroxybutyrate in our experiments. It can be noted that cells are able to use only the D-isomer of $\beta$ hydroxybutyrate. Therefore, it is expected that only $50 \%$ of the provided $\beta$-hydroxybutyrate will be utilized in our experiments (Tsai et al., 2006; Holmgren et al., 2010; Mukhtarov et al., 2011). However, it can be noted that the L-isomer also can have some biological activity, for instance, it has the same antioxidant properties as D-isomer (Julio-Amilpas et al., 2015). Moreover, the presence of the L-isomer can reduce the utilization of the D-isomer as it will compete for transport, thus leading to an underestimation of the effects observed.

In the present study, we have found that exposing synaptosomes to glucose-free medium containing $\beta$-hydroxybutyrate or pyruvate did not lead to irreversible damage following 
plasma membrane depolarization. It was shown previously that synaptosomes are able to survive using pyruvate alone as energy substrate (Kauppinen and Nicholls, 1986; Tarasenko et al., 2006). Also, it is established that relatively short (up to 30 minutes) incubation period of synaptosomes without any source of energy leads to a decrease but not to the full dissipation of plasma and mitochondrial membrane potentials (Kauppinen and Nicholls, 1986 Hrynevich et al., 2015). Switching synaptosomes from glucose to $\beta$-hydroxybutyrate as their main energy substrate does not lead to changes in plasma membrane potential. In presence of pyruvate instead, a slight decrease of this parameter was observed. It was shown previously that addition of ketone bodies or pyruvate to the incubation medium stimulated ATP synthesis (Tarasenko et al., 2006; Kim et al., 2010). These observations support the point of view that neurons, or at least their synaptic components, are able to use nonglycolytic energy substrates to maintain this essential parameter for their signalling function. While no significant depolarization of the plasma membrane was seen in presence of $\beta$-hydroxybutyrate, we also failed to observe any hyperpolarization as it was shown by Holmgren et al. (2010). In fact, activation of $\mathrm{K}_{\text {АтP }}$ channels has been proposed as one mechanism to explain the beneficial effects of the ketogenic diet on excitability (Gimenez-Cassina et al., 2012; Gano et al., 2014; Lund et al., 2015). Our results seem to rule out this possibility for synapses. Indeed, synaptosomes express this type of potassium channel and they normally exhibit plasma membrane hyperpolarization when these channels are directly activated (Bancila et al., 2004).

The fluorescent dye acridine orange can be used to monitor synaptic vesicle recycling. This dye is concentrated in acid organelles where its fluorescence is quenched, mainly in synaptic vesicles, when entrapped into synaptosomes. Dissipation of the $\mathrm{pH}$ gradient, either in case of exocytosis, following a treatment with a proton ionophore or with v-ATPase inhibitors, leads to dequenching of acridine orange and an increase in fluorescence intensity. In the case of exocytosis, it subsequently induces a compensatory endocytosis which can be monitored by following the fluorescence decrease (Zoccarato et al., 1999; Waseem et al., 2005; Hrynevich et al., 2015). A typical response of acridine orange fluorescence to potassium-induced depolarization in synaptosomes is depicted in Figure 2a. Using this methodological approach, we have shown that synaptic vesicle recycling can be similarly sustained when the energetic substrate is switched from glucose to $\beta$-hydroxybutyrate. However, in presence of $\beta$-hydroxybutyrate alone, exocytosis is slightly stimulated and the compensatory endocytosis is reduced compared to the response obtained with glucose. Addition of $\beta$-hydroxybutyrate to glucose-containing incubation medium does not influence 
endocytosis however, which rules out a direct influence of ketone bodies on this process. Use of pyruvate as sole energy substrate led to the same effects as in presence of $\beta$ hydroxybutyrate alone. These results suggest that the reason for endocytosis inhibition with nonglycolytic substrates lies in the glycolytic metabolism of glucose compared to nonglycolytic energy substrates. Supporting our finding, it was reported recently that replacement of glucose by pyruvate in cultured hippocampal neurons leads to a small but statistically significant inhibition of endocytosis (Pathak et al., 2015). Also it was found that endocytosis is the most sensitive process in neuronal presynaptic endings to a decrease of ATP levels (Rangaraju et al., 2014).

In our experiments, the reduction of acridine orange fluorescence decrease in presence of $\beta$-hydroxybutyrate can be explained by at least two possibilities: 1) Inhibition of synaptic vesicle budding from the plasma membrane. This means that bona fide endocytosis is reduced 2) A decrease in v-ATPase activity which is responsible for reacidification of synaptic vesicles after compensatory endocytosis (Zoccarato et al., 1999; Toei et al., 2010). The vATPase is an energy-consuming enzyme by definition and its ATP supply might be dependent on the energy substrate used.

Inhibition of endocytosis can be further studied using the styryl dye FM2-10 or its analogue FM1-43, which is widely employed for neuronal imaging. However, in the case of synaptosomes, FM2-10 provides better responses (Cousin and Robinson, 2000; Fedorovich et al., 2005). In contrast to acridine orange, styryl dyes are able to reveal the processes of fusion and budding of lipid bilayers (Zoccarato et al., 1999; Cousin and Robinson, 2000). Indeed, FM2-10 reports exocytosis by fluorescence decrease (Cousin and Robinson, 2000; Waseem et al., 2005). After synaptosomes were loaded with the FM2-10 dye in control glucosecontaining incubation medium and in $\beta$-hydroxybutyrate-containing medium, dye release was then induced by potassium-induced depolarization in control incubation medium only. Under these conditions, reduction of endocytosis was reproducible. Furthermore, it was even more substantial than revealed with acridine orange fluorescence. Also we investigated exocytosis monitored by this dye. Synaptosomes were loaded in control conditions and the dye was released in gluocose or $\beta$-hydroxybutyrate-containing medium. We have shown that exocytosis was also inhibited, however such an inhibition was less pronounced than for endocytosis. Exocytosis was reduced by half; endocytosis was reduced by two thirds (Figs. $3 b, c)$. Results with FM2-10 were significantly more pronounced than in the case of acridine orange (Figs 2b,c, 3b, c). Peak height of acridine orange fluorescence (Fig. 2a) is most likely 
resulted from equilibrium between exo- and endocytosis. Strong inhibition of compensatory endocytosis masks reduction of exocytosis. Vice versa, inhibition of exocytosis partly masks reduction of endocytosis. However, we were able to clearly distinguish between exocytosis and endocytosis by using the FM2-10 dye. Thus, our results clearly supports the concept that switching synaptosomes from glucose to nonglycolytic energy substrates reduces bone fide endocytosis and leads to misbalance between endocytosis and exocytosis. Also we have shown that replacement of glucose by ketone bodies can directly inhibit exocytosis.

Recently it was found that endocytosis is also a very sensitive process to energetic deficit in synaptic terminals of hippocampal neurons (Rangaraju et al., 2014; Pathak et al., 2015). Furthermore, vesicle budding from the plasma membrane is significantly more inhibited than reacidification in this condition (Pathak et al., 2015). Our results also suggest that vesicle budding could be a target for several bioenergetic disturbances in synapses.

Nonglycolytic energy substrates are able to support mitochondrial activity, but not the pentose phosphate pathway which is the main source for NADPH in a cell (White and Venkatesh, 2011; Rodriguez-Rodriguez, 2013). However, 6-aminonicotinamide (1 mM), an inhibitor of the pentose phosphate pathway (Osada-Oka et al., 2010), does not inhibit endocytosis monitored with the fluorescent dye acridine orange (data not shown). Thus, it is unlikely that activity of the pentose phosphate pathway per se, or the supply of NADPH, is the key factor explaining the difference between glucose and nonglycolytic substrates. It was shown that glycolytic enzymes are enriched in synaptic vesicles (Ikemoto et al., 2003). Furthermore, synaptic vesicle loading with neurotransmitters is dependent on local glycolytic activity rather than on total ATP content in neurons (Ikemoto et al., 2003). It was established that high local ATP concentration created by glycolytic enzymes is crucial for regulation of different plasma membrane channels and transporters (Dhar-Chowdhury et al, 2007). We hypothesize that endocytosis might require such ATP microdomains. These potential microdomains would be created most likely by glycolytic enzyme activities. However, when synapses are exposed solely to nonglycolytic energy substrates, they might be able to maintain a certain activity, but the disappearance of these ATP microdomains would result in a reduction of endocytosis.

We have observed that the $\mathrm{pH}$ gradient of synaptic vesicles was not changed after exposing synaptosomes to $\beta$-hydroxybutyrate as energy substrate rather than glucose. This could be explained by the fact that, as we have shown previously, this parameter although being directly regulated by the v-ATPase is the most resistant to energy deficit in neuronal 
presynaptic endings (Hrynevich et al., 2015). In order to confirm this observation in intact preparation, we investigated the capacity of $\beta$-hydroxybutyrate to maintain the synaptic vesicle membrane potential in neurons in vitro. Cultured mouse cortical neurons expressing the genetically encoded sensor synaptopHluorin were used for that purpose (Miesenbock et al., 1998; Burron, 2006). This approach is similar to the one relying on acridine orange's response. SynaptopHluorin is the synaptic vesicle protein synaptobrevin fused to the green fluorescent protein. This construct is expressed in synaptic vesicles where its fluorescence is quenched at low $\mathrm{pH}$. Exocytosis or treatment with a proton ionophore leads to dequenching with subsequent fluorescence increase. Synaptic boutons expressing synaptopHluorin are able to respond to the proton ionophore FCCP by exhibiting fluorescence increase. Incubation of neurons for one hour in an incubation medium without energy substrates leads to almost full dissipation of synaptic vesicles $\mathrm{pH}$ gradient. Addition of $\beta$-hydroxybutyrate to the incubation medium completely restored this parameter. Our results thus confirmed that synapses in situ can use also ketone bodies at least for maintaining some key biophysical parameters of synaptic vesicles.

\section{CONCLUSION}

We have shown that ketone bodies such as $\beta$-hydroxybutyrate are able to support synaptic vesicle recycling. However ketone bodies when fueling the synaptic vesicle cycle, modify it. Indeed, the ratio between endocytosis and exocytosis is decreased. Which physiological consequences can be expected ? The decrease of the ratio between exocytosis and compensatory endocytosis is supposed to deplete the synaptic vesicle pool during intensive stimulation, for instance following an epileptic burst. If our hypothesis is correct, this depletion might contribute to terminate seizures. In support of these suggestions, it was shown that inhibition of endocytosis caused short-term synaptic depression (Hua et al., 2013). Also direct inhibition of exocytosis (Fig. 3c) can be involved in the anticonvulsant activity of the ketogenic diet. Therefore, modification of the synaptic vesicle cycle in the case of neurons fueled with nonglycolytic energy substrates instead of glucose could provide the mechanism explaining the anti-seizure action of the ketogenic diet. 
Acknowledgements. This work was supported by the Bielorussian Republican Foundation of Basic Investigation (grant B13-066) and the Swiss National Science Foundation (grant IZKOZ3_147590).

Funding agencies had no involvement in study design, in the collection, analysis and interpretation of data, in the writing of the report, and in the decision to submit the article for publication.

We thank Prof. G. Miesenbock (University of Oxford, Oxford, UK) for synaptopHluorin plasmid, Dr. L. Bozzo (Cell Imaging Facility, University of Lausanne, Lausanne, Switzerland) and Dr. J.-Y. Chatton (Cell Imaging Facility, University of Lausanne, Lausanne, Switzerland) for their help with fluorescence microscopy. 


\section{REFERENCES}

Attwell, D., Gibb, A., 2005. Neuroenergetic and the kinetic design of excitatory synapses. Nature Rev. Neurosci. 6, 841-849.

Attwell, D., Laughlin, S.B., 2001. An energy budget for signaling in the grey matter of the brain. J. Cereb. Blood Flow Metab. 21, 1133-1145.

Ballaban-Gil, K., Callahan, C., O’Dell, C., Pappo, M., Moshe, S., Shinnar, S., 1998. Complications of the ketogenic diet. Epilepsia 39, 744-748.

Bancila, V., Nikonenko, I., Dunant, Y., Bloc, A., 2004. Zinc inhibits glutamate release via activation of pre-synaptic $\mathrm{K}_{\mathrm{ATP}}$ channels and reduces ischaemic damage in rat hippocampus. J. Neurochem. 90, 1243-1250.

Bergersen, L.H., 2014. Lactate transport and signaling in the brain: potential therapeutic targets and roles in body-brain interaction. J. Cereb. Blood Flow Metab. 35, 176-185.

Brewer, G.J., Torricelli, J.R., Evege, E.K., Price, P.J., 1993. Optimized survival of hippocampal neurons in B27-supplemented neurobasal, a new serum-free medium combination. J. Neurosci. Res. 35, 567-576.

Brewer, G.J., 1995. Serum-free B27/neurobasal medium supports differentiated growth of neurons from the striatum, substantia nigra, septum, cerebral cortex, cerebellum, and dentate gyrus. J. Neurosci. Res. 42, 674-683.

Burrone, J., Li, Z., Murthy, V., 2006. Studying vesicle cycling in presynaptic terminals using the genetically encoded probe synaptopHluorin. Nat. Protocols 1, 2970-2978.

Castro, M.A., Beltran, F.A., Brauchi, S., Concha, I.I., 2009. A metabolic switch in brain: glucose and lactate metabolism modulation by ascorbic acid. J. Neurochem. 110, 423-440.

Cousin, M.A., Robinson, P.J., 1999. Two mechanisms of synaptic vesicle recycling in rat brain synaptosomes. J. Neurochem. 75, 1645-1653.

Dedkova, E.N., Blatter, L.A., 2014. Role of $\beta$-hydroxybutirate, its polymer poly- $\beta$ hydroxybutirate and inorganic polyphosphate in mammalian health and disease. Front. Physiol. 5, 260.

Dhar-Chowdhury, P., Malester, B., Rajacic, P., Rajacic, Coetzee, W.A., 2007. The regulation of ion channels and transporters by glycolytically derived ATP. Cell. Mol. Life Sci. 64, 3069-3083. 
Elliott, K.A.C., Greig, M.E., Benoy, M.P., 1937. The metabolism of lactic and pyruvic acids in normal and tumour tissues. III Rat liver, brain and testis. Biochem. J. 31, 1003-1020.

Fedorovich, S.V., Waseem, T.V., Lavrukevich, T.V., Konev, S.V. 2005. Role of calcium in exocytosis induced by hypotonic swelling. Ann. NY Acad. Sci. 1048, 337-340.

Gano, L.B., Patel, M., Rho, J.M., 2014. Ketogenic diets, mitochondria, and neurological diseases. J. Lipid Res. 55, 2211-2228.

Garbow, J.R., Doherty, J.M., Schugar, R.C., Travers, S., Weber, M.L., Wentz, A.E., Ezenwajiaku, N., Cotter, D.G., Brunt, E.M., Crawford, P.A., 2011. Hepatic steatosis, inflammation, and ER stress in mice maintained long term on a very low-carbohydrate ketogenic diet. Am. J. Physiol. Gastrointest. Liver Physiol. 300, G956-G967.

Giminez-Cassina, A., Martinez-Francoi,s J.R., Fisher, J.K., Szlyk, B., Polak, K., Wiwczar, J., Tanner, G.R., Lutas, A., Yellen, G., Danial, N.N., 2012. BAD-dependent regulation of fuel metabolism and $\mathrm{K}_{\mathrm{ATP}}$ channel activity confers resistance to epileptic seizures. Neuron 74, 719-730.

Hajos, F., 1975. An improved method for the preparation of synaptosomal fractions in high purity. Brain Res. 93, 485-489.

Harris, J.J., Jolivet, R., Attwell, D., 2012. Synaptic energy use and supply. Neuron 75, 762777.

Hawkins, R.A., Williamson, D.H., Krebs, H.A., 1971. Ketone-body utilization by adult and suckling rat brain in vivo. Biochem. J. 122, 13-18.

Holmgren, C.D., Mukhtarov, M., Malkov, A.E., Popova, I.Y., Bregestovski, P., Zilberter, Y., 2010. Energy substrate availability as a determinant of neuronal resting potential, GABA signaling and spontaneous network activity in the neonatal cortex in vitro. J. Neurochem. $112,900-912$.

Hrynevich, S.V., Pekun, T.G., Waseem, T.V., Fedorovich, S.V., 2015. Influence of glucose deprivation on membrane potentials of plasma membranes, mitochondria and synaptic vesicles in rat brain synaptosomes. Neurochem. Res. 40:1188-1196.

Hua, Y., Woehler, A., Kahms, M., Haucke, V., Neher, E., Klingauf, J., 2013. Blocking endocytosis enhances short-term synaptic depression under conditions of normal availability of vesicles. Neuron 80, 343-349. 
Ikemoto, A., Bole, D.G., Ueda, T., 2003. Glycolisis and glutamate accumulation into synaptic vesicles. Role of glyceraldehyde phosphate dehydrogenase and 3-phosphoglycerate kinase. J. Biol. Chem. 278, 5929-5940.

Izumi, Y., Ishii, K., Katsuki, H., Benz, A.M., Zorumski, C.F., 1998. $\beta$-hydroxybutirate fuels synaptic function during development. Histological and physiological evidence in rat hippocampal slices. J. Clin. Invest. 101, 1121-1132.

Juge, N., Gray, J.A., Omote, H., Miyaji, T., Inoue, T., Hara, C., Uneyama, H., Edwards, R.H., Nicoll, R.A., Moriyama, Y., 2010. Metabolic control of vesicular glutamate transport and release. Neuron 68, 99-112.

Julio-Amilpas, A., Montiel, T., Soto-Tinoco, E., Geronimo-Olvera, C., Massieu, L., 2015. Protection of hypoglycemia-induced neuronal death by $\beta$-hydroxybutirate involves the preservation of energy levels and decreased production of reactive oxygen species. $\mathrm{J}$. Cereb. Blood Flow Metab. 35, 851-860.

Kauppinen, R.A., Nicholls, D.G., 1986. Synaptosomal bioenergetics. The role of glycolisis, pyruvate oxidation and responses to hypoglycaemia. Eur. J. Biochem. 158, 159-165.

Kety, S.S., Schmidt, C.F., 1946. The effects of active and passive hyperventilation on cerebral blood flow, cerebral oxygen consumption, cardiac output, and blood pressure of normal young men. J. Clin. Invest. 25, 107-119.

Kim, D.Y., Vallejo, J., Rho, J.M., 2010. Ketones prevent synaptic dysfunction induced by mitochondrial respiratory complex inhibitors. J. Neurochem. 114, 130-141.

Lowry, O., Rosenbrough, H., Farr, H., Randall, R., 1951. Protein measurements with Folin reagent. J. Biol. Chem. 193, 265-279.

Lund, T.M., Ploug, K.B., Iversen, A., Jensen, A.A., Jansen-Olesen, I., 2015. The metabolic impact of $\beta$-hydroxybutirate on neurotransmission: reduced glycolysis mediates changes in calcium responses and $\mathrm{K}_{\mathrm{ATP}}$ channel receptor sensitivity. J. Neurochem. 132:520-531.

McKenna, M.C., Tildon, J.T., Stevenson, J.H., Hopkins, I.B., Huang, X., Couto, R., 1998. Lactate transport by cortical synaptosomes from adult rat brain: characterization of kinetics and inhibitor specificity. Dev. Neurosci. 20, 300-309.

McNally, M.A., Hartman, A.L., 2012. Ketone bodies in epilepsy. J. Neurochem. 121, 28-35.

Miesenbock, G., De Angelis, D.A., Rothman, J.E., 1998. Vizualizing secretion and synaptic transmission with pH-sensitive green fluorescent proteins. Nature 394, 192-195. 
Morgenthaler, F.D., Kraftsik, R., Catsikas, S., Magistretti, P.J., Chatton, J.-Y., 2006. Glucose and lactate are equally effective in energizing activity-dependent synaptic vesicle turnover in purified cortical neurons. Neuroscience 141, 157-165.

Mukhtarov, M., Ivanov, A., Zilberter, Y., Bregestovski, P., 2011. Inhibition of spontaneous network activity in neonatal hippocampal slices by energy substrates is not correlated with intracellular acidification. J. Neurochem. 116, 316-321.

Osada-Oka, M., Hashiba, Y., Akiba, S., Imaoka, S., Sato, T., 2010. Glucose is necessary for stabilization of hypoxia-inducible factor-1 $\alpha$ under hypoxia: contribution of the pentose phosphate pathway to this stabilization. FEBS Lett. 584, 3073-3079.

Paoli, A., Bianco, A., Damiani, E., Bosco, G., 2014. Ketogenic diet in neuromuscular and neurodegenerative diseases. BioMed. Res. Int 2014, 474296.

Pathak, D., Shields, L., Mendelsohn, B.A., Haddad, D., Lin, W., Gerencser, A.A., Kim, H., Brand, M.D., Edwards, R.H., Nakamura, K., 2015. The role of mitochondrially derived ATP in synaptic vesicle recycling. J. Biol. Chem. 290, 22325-22336.

Pellerin, L., Magistretti, P.J., 2012. Sweet sixteen for ANLS. J. Cereb. Blood Flow Metab. 32, $1152-1166$.

Peterman, M.G., 1924. The ketogenic diet in the treatment of epilepsy: a preliminary report. Am. J. Dis. Child 28, 28-33.

Pierre, K., Pellerin, L., 2005. Monocarboxylate transporters in the central nervous system: distribution, regulation and function. J. Neurochem. 94, 1-14.

Rangaraju, V., Calloway, N., Ryan, T.A., 2014. Activity-driven local ATP synthesis is required for synaptic function. Cell 156, 825-835.

Robinet, C., Pellerin, L., 2010. Brain-derived neurotrophic factor enhances the expression of the monocarboxylate transporter 2 through translation activation in mouse cultured cortical neurons. J. Cereb. Blood Flow Metab. 30, 286-298.

Rodriguez-Rodriguez, P., Almeida, A., Bolanos, J.P., 2013. Brain energy metabolism in glutamate-receptor activation and excitotoxicity: role for APC/C-Cdh1 in the balance glycolysis/penthose phosphate pathway. Neurochem. Int. 62, 750-756.

Rolleston, F.S., Newsholme, E.A., 1967. Effects of fatty acids, ketone bodies, lactate and pyruvate on glucose utilization by guinea-pig cerebral cortex slices. Biochem. J. 104, 519523. 
Sampath, A., Kossoff, E.H., Furth, S.L., Pyzik, P.L., Vining, E.P., 2007. Kidney stones ant ketogenic diet: risk factors and prevention. J. Child Neurol. 22, 375-378

Stafstrom, C.E., Rho, J.M., 2012. The ketogenic diet as a treatment paradigm for diverse neurological disorders. Front. Pharmacol. 3, 59.

Südhof, T.C., 2004. The synaptic vesicle cycle. Annu. Rev. Neurosci. 27, 509-547.

Südhof, T.C., 2013. Neurotransmitter release: the last millisecond in the life of a synaptic vesicle. Neuron 80, 675-690.

Tarasenko, A.S., Linetska, M.V., Storchak, L.G., Himmelreich, N.H., 2006. Effectiveness of extracellular lactate/pyruvate for sustaining synaptic vesicle proton gradient generation and vesicular accumulation of GABA. J. Neurochem. 99, 787-796.

Toei, M., Saum, R., Forgac, M., 2010. Regulation and isoform function of the V-ATPases. Biochemistry 49, 4715-4723.

Tsai, Y.C., Chou, Y.C., Wu, A.B., Hu, C.M., Chen, C.Y., Chen, F.A., Lee, J.A., 2006. Stereoselective effects of 3-hydroxybutirate on glucose utilization of rat cardiomyocytes. Life Sci. 78, 1385-1391.

Waseem, T.V., Rakovich, A.A., Lavrukevich, T.V., Konev, S.V., Fedorovich, S.V., 2005. Calcium regulates the mode of exocytosis induced by hypotonic shock in isolated neuronal presynaptic endings. Neurochem. Int. 46, 235-242.

Waseem, T.V., Kolos, V.A., Lapatsina, L.P., Fedorovich, S.V., 2007. Hypertonic shrinking but not hypotonic swelling increases sodium concentration in rat brain synaptosomes. Brain Res. Bull. 73, 135-142.

Waseem, T.V., Fedorovich, S.V., 2010. Presynaptic glycine receptors influence plasma membrane potential and glutamate release. Neurochem. Res. 35, 1188-1195.

Wilhelm, B.G., Mandad, S., Truckenbrodt, S., Kröhnert, K, Schäfer, C., Rammner, B., Koo, S.J., Claßen, G.A., Krauss, M., Haucke, V., Urlaub, H., Rizzoli, S.O., 2014. Composition of isolated synaptic boutons reveals the amounts of vesicle trafficking proteins. Science 344, 1023-1028.

White, H., Venkatesh, B., 2011. Clinical review: ketones and brain injury. Critical Care 15, 219.

Zoccarato, F., Cavallini, L., Alexandre, A., 1999. The $\mathrm{pH}$-sensitive dye acridine orange as a tool to monitor exocytosis/endocytosis in synaptosomes. J. Neurochem. 72, 625-633. 
FIGURE LEGENDS

Figure 1. Effect of nonglycolytic energy substrates on plasma membrane potential in rat brain synaptosomes measured with DiSC3(5) fluorescence. Glucose in the incubation medium was replaced by either $8 \mathrm{mM}$ DL- $\beta$-hydroxybutyrate (BHB) or by $4 \mathrm{mM}$ pyruvate (PYR). Each bar represents DiSC3(5) fluorescence increase within 4 minutes after addition of $150 \mathrm{mM} \mathrm{KCl}$. Data are presented as mean \pm SEM of 8 experiments for BHB and of 11 experiments for Pyr. The 100\% level corresponds to fluorescence increase in glucosecontaining control medium. ${ }^{* *} \mathrm{P} \leq 0.01$ vs. $100 \%$.

Figure 2. Effect of nonglycolytic energy substrates on synaptic vesicle recycling in rat brain synaptosomes measured with acridine orange fluorescence. A) Kinetic of acridine orange fluorescence after $\mathrm{KCl}(60 \mathrm{mM})$ addition. $\mathrm{KCl}$ was added when indicated. Curves represent 9 independent experiments. B) Effect of nonglycolytic energy substrates on acridine orange fluorescence increase (exocytosis) after potassium depolarization. Each bar represents maximal increase of acridine orange fluorescence after addition of $60 \mathrm{mM} \mathrm{KCl}$ for each substrate or combination (see below). Data presented are mean \pm SEM of 9 experiments. The $100 \%$ level corresponds to fluorescence increase in glucose-containing control medium. $* \mathrm{P} \leq 0.05$ vs. $100 \%$. C) Effect of nonglycolytic energy substrates on acridine orange fluorescence decrease (endocytosis) after potassium depolarization. Each bar represent decrease of acridine orange fluorescence after maximal increase when $60 \mathrm{mM} \mathrm{KCl}$ for each substrate or combination (see below). Data presented are mean \pm SEM of 9 experiments. The $100 \%$ level corresponds to fluorescence decrease in glucose-containing control medium. $* * \mathrm{P} \leq 0.01$ vs. $100 \%$. D) Effect of nonglycolytic energy substrates on endocytosis/exocytosis ratio. Each bar represents the ratio between decrease of acridine orange fluorescence after maximal increase in case of potassium-induced depolarization and maximal increase of acridine orange fluorescence after addition of $60 \mathrm{mM} \mathrm{KCl}$ for each substrate or combination (see below). Data presented are mean SEM of 9 experiments. ${ }^{* *} \mathrm{P} \leq 0.01$ vs. $100 \%$. Con (Control), glucose-containing control incubation medium; BHB ( $\beta$-hydroxybutyrate), glucose in incubation medium was replaced by $8 \mathrm{mM}$ DL- $\beta$-hydroxybutyrate; Pyr (Pyruvate), glucose in incubation medium was replaced by $4 \mathrm{mM}$ pyruvate; $\mathrm{BHB}+\mathrm{Glu}$ ( $\beta$ hydroxybutyrate + Glucose) incubation medium contained $4 \mathrm{mM}$ glucose $+4 \mathrm{mM}$ DL- $\beta-$ hydroxybutyrate. 
Figure 3. Effect of $\beta$-hydroxybutyrate on endocytosis in rat brain synaptosomes measured with the fluorescent dye FM2-10. A) Kinetic of FM2-10 fluorescence decline, caused by potassium-induced depolarization. Sixty $\mathrm{mM} \mathrm{KCl}$ was added when indicated. Each curve represents 5 independent experiments. Con - synaptosomes were loaded with the dye in glucose-containing medium. BHB - synaptosomes were loaded with the dye in $\beta$ hydroxybutyrate- containing medium. B) Quantitative assessment of endocytosis. Synaptosomes were loaded with the dye in control glucose-containing or $\beta$-hydroxybutyrate containing incubation medium. Dye was released in control glucose-containing medium. Each bar represents FM2-10 fluorescence decrease within 5 minutes after addition of $60 \mathrm{mM} \mathrm{KCl}$. C) Quantitative assessment of exocytosis. Dye was released from synaptosomes in control glucose-containing or $\beta$-hydroxybutyrate containing incubation medium. Synaptosomes were loaded with the dye in control glucose-containing medium. Each bar represents FM2-10 fluorescence decrease within 5 minutes after addition of $60 \mathrm{mM} \mathrm{KCl}$. Data presented are mean \pm SEM of 5 experiments. The $100 \%$ level corresponds to fluorescence decrease in glucose-containing control medium. ${ }^{*} \mathrm{P} \leq 0.01$ vs. $100 \%$. Con (Control), glucose-containing control incubation medium; BHB ( $\beta$-hydroxybutyrate) glucose in the incubation medium was replaced by $8 \mathrm{mM}$ DL- $\beta$-hydroxybutyrate

Figure 4. Effect of $\beta$-hydroxybutyrate on $\mathrm{pH}$ gradient of synaptic vesicles in rat brain symaptosmes revealed with acridine orange fluorescence. A) Kinetic of acridine orange fluorescence after bafilomycin A1 (100 nM) addition. Bafilomycin (Baf) was added when indicated. Curves represent 7 independent experiments. B) Quantitative assessment of bafilomycin-induced, acridine orange fluorescence increase reflecting synaptic vesicle $\mathrm{pH}$ gradient. Each bar represents acridine orange fluorescence increase within 5 minutes after addition of $100 \mathrm{nM}$ bafilomycin. Data presented are mean \pm SEM of 7 experiments. The $100 \%$ level corresponds to fluorescence increase in glucose-containing control medium. Con (Control), glucose-containing control incubation medium; BHB ( $\beta$-hydroxybutyrate), glucose in incubation medium was replaced by $8 \mathrm{mM}$ DL- $\beta$-hydroxybutyrate

Figure 5. Effect of $\beta$-hydroxybutyrate on $\mathrm{pH}$ gradient of synaptic vesicles in cortical neurons assessed with synaptoHluorin. A) Kinetic of synaptopHluorin response upon FCCP treatment. FCCP $(10 \mu \mathrm{M})$ was applied when indicated. Curves were recorded in normal glucose-containing incubation medium. B) Kinetic of synaptopHluorin response upon FCCP 
treatment. FCCP $(10 \mu \mathrm{M})$ was applied when indicated. Curves were recorded in BHBcontaining incubation medium. C) Kinetic of synaptopHluorin response upon FCCP treatment. FCCP $(10 \mu \mathrm{M})$ was applied when indicated. Curves were recorded in incubation medium without energy substrates. D) Quantitative assessment of synaptic vesicle $\mathrm{pH}$ gradient following incubation with or without energy substrates. Each bar represents the synaptopHluorin fluorescence increase within 3 minutes after FCCP application. Data presented are mean \pm SEM of independent measurements in 22 synaptic boutons in the case of control glucose-containing incubation medium, in 41 synaptic boutons in the case of DL- $\beta$ hydroxybutyrate containing incubation medium, and in 8 synaptic boutons in the case of incubation medium without energetic substrates. Two coverslips were imaged for each condition. The $100 \%$ level corresponds to fluorescence increase in glucose-containing control medium. ${ }^{*} \mathrm{P} \leq 0.01$ vs. $100 \%$. Con (Control) glucose-containing control incubation medium; BHB ( $\beta$-hydroxybutyrate) glucose in incubation medium was replaced by $8 \mathrm{mM}$ DL- $\beta$ hydroxybutyrate; GW (Glucose Withdrawal), glucose was withdrawn from the incubation medium. 
Fig. 1

\section{BHB}

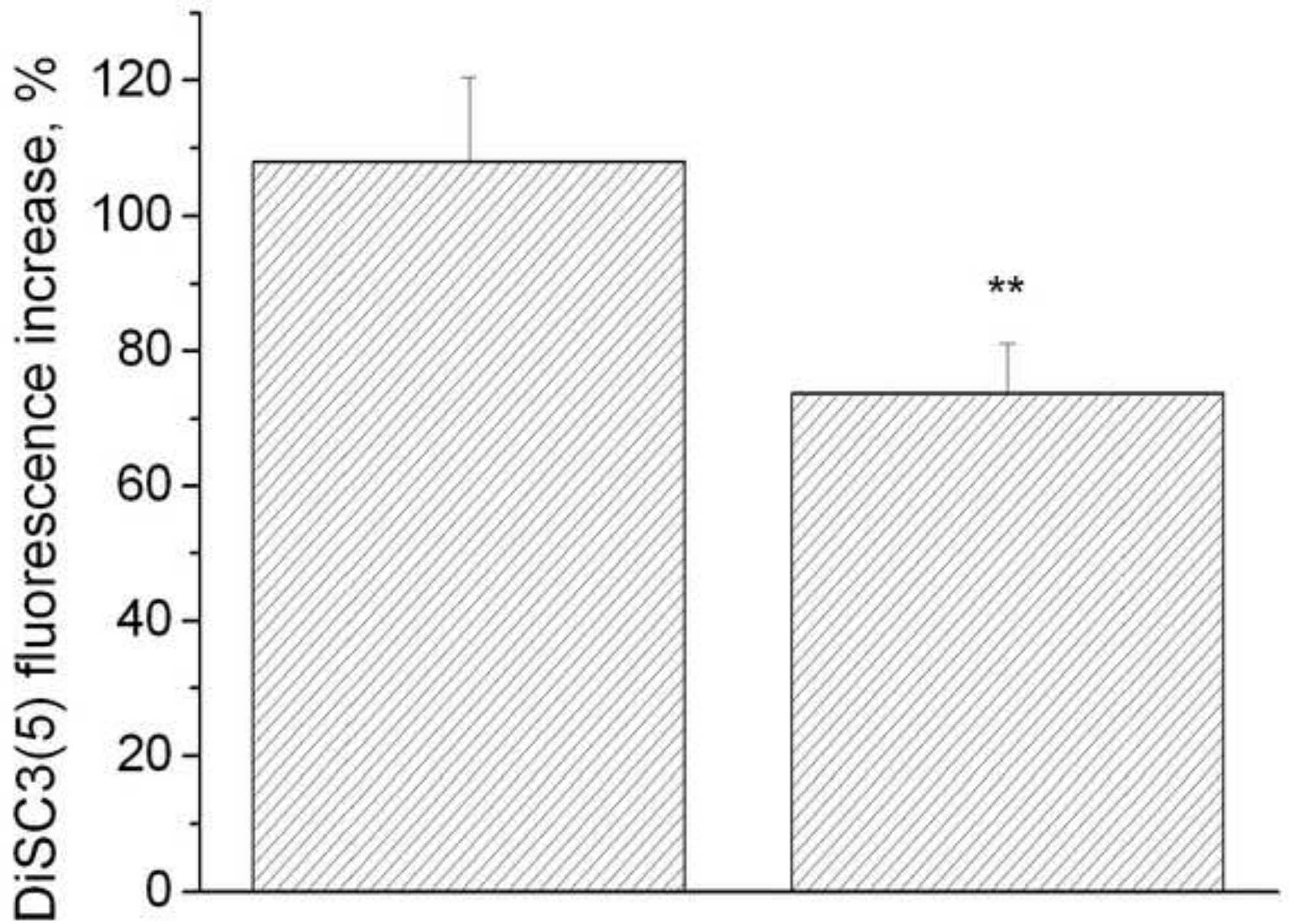




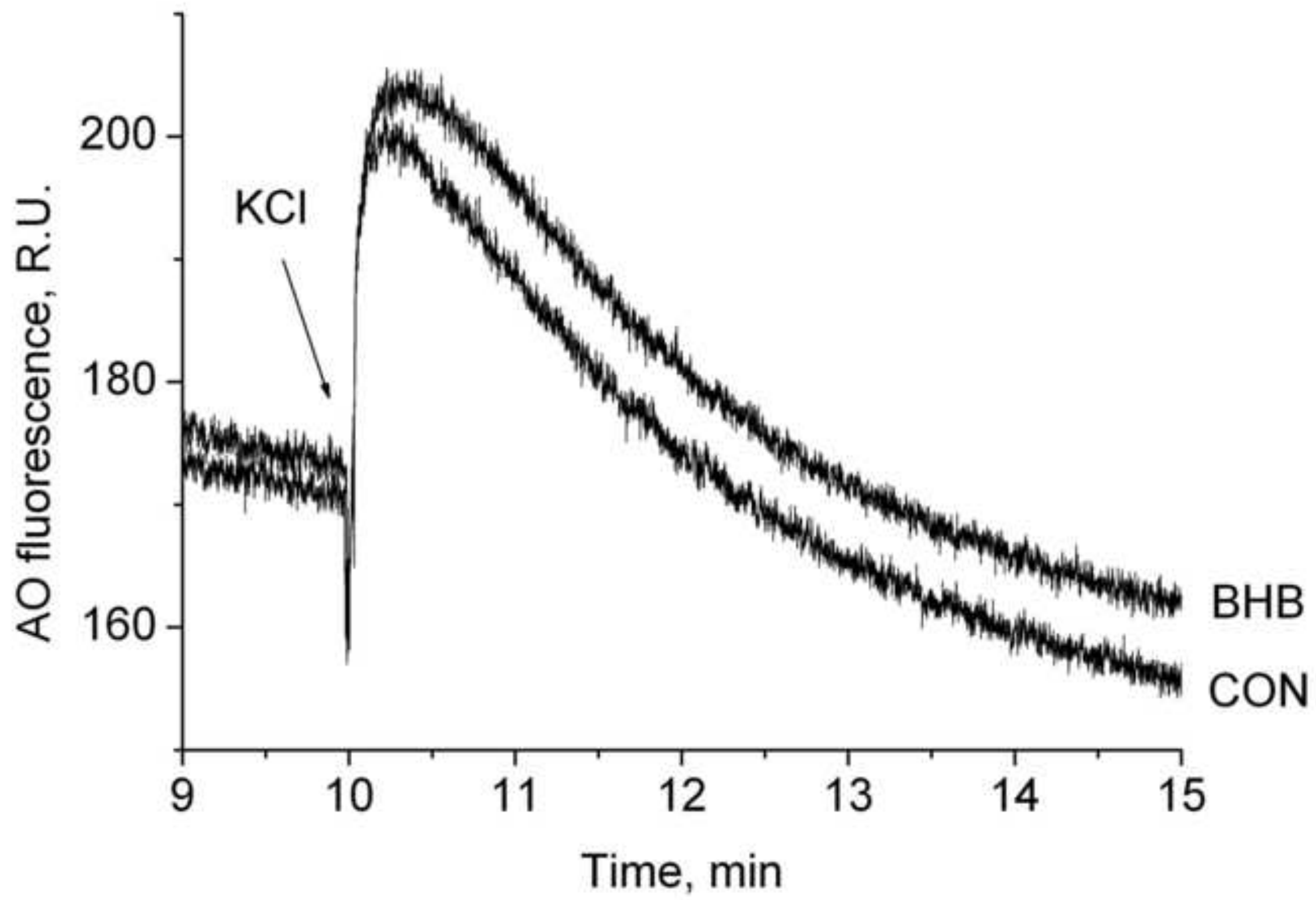


Con BHB BHB+Glu Pyr

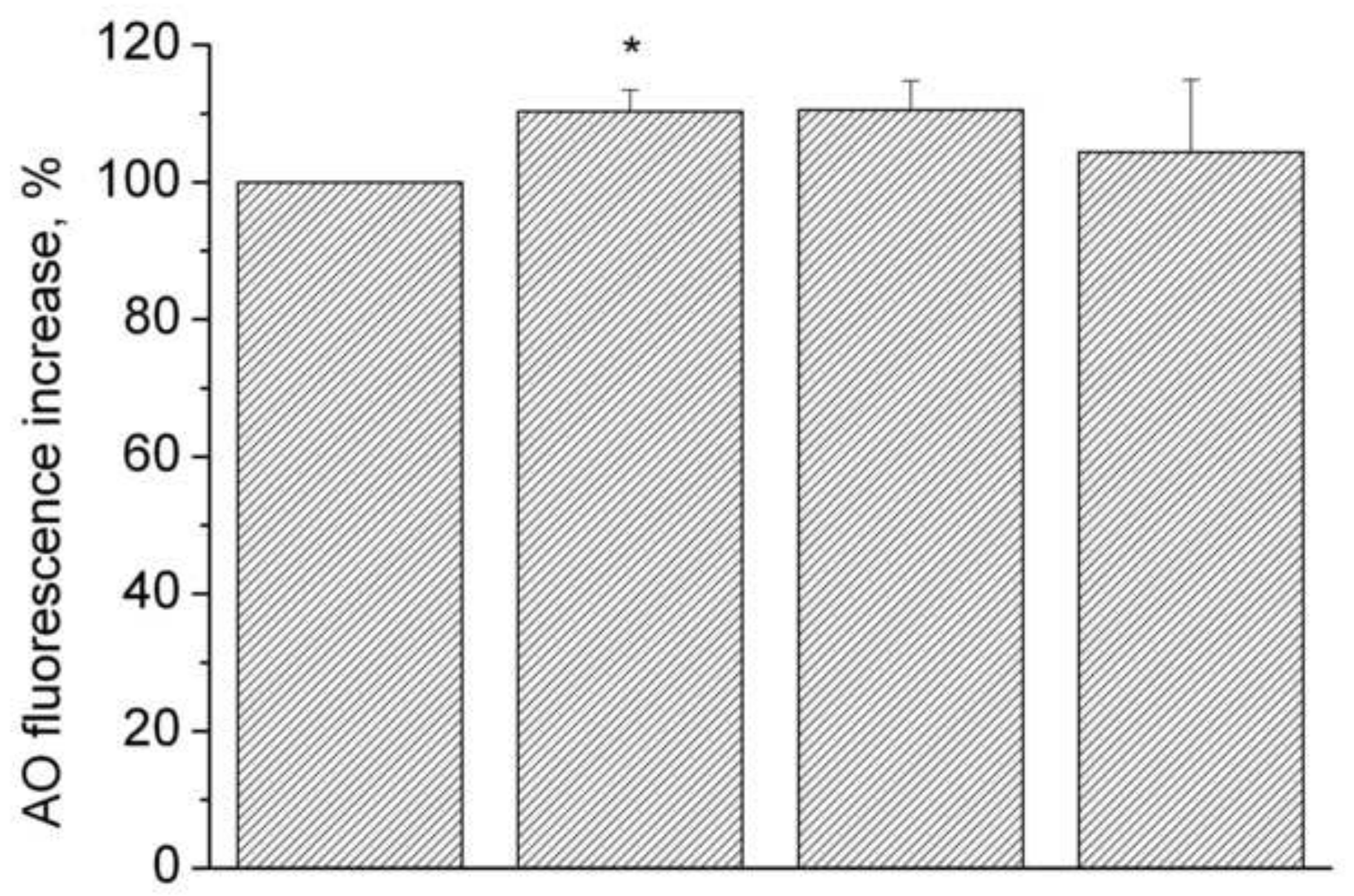




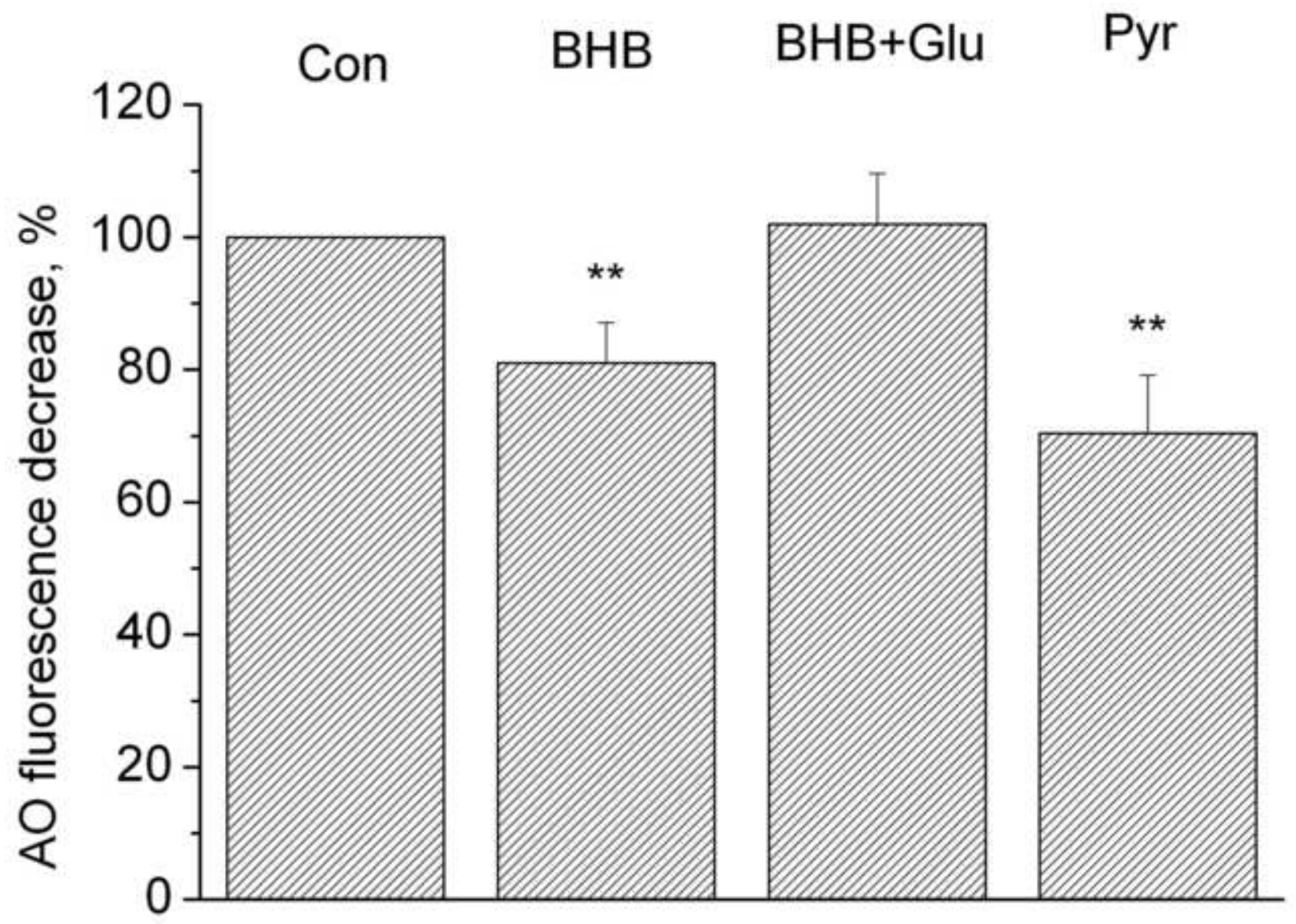


Con BHB BHB+Glu Pyr

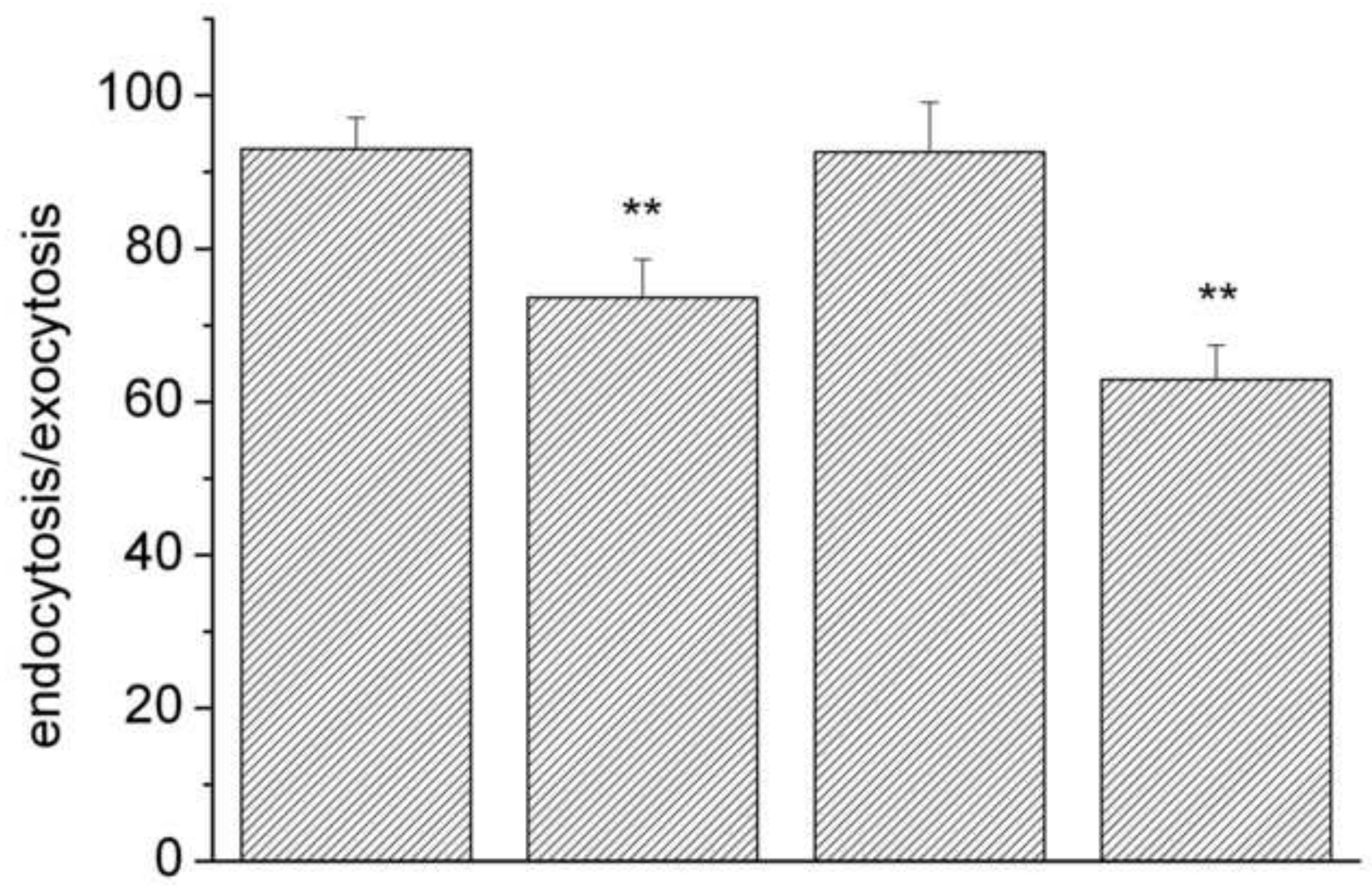




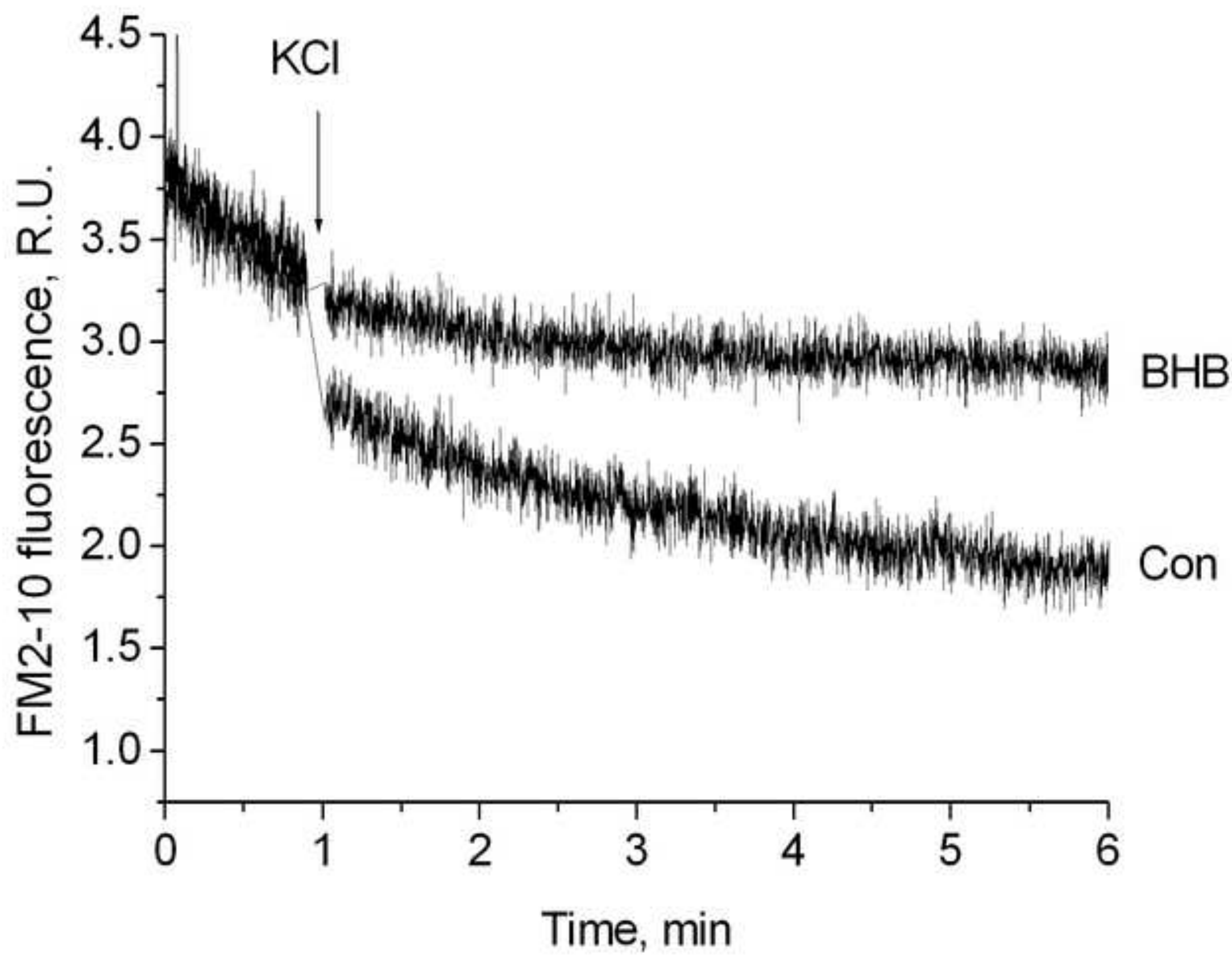


Con

\section{BHB}

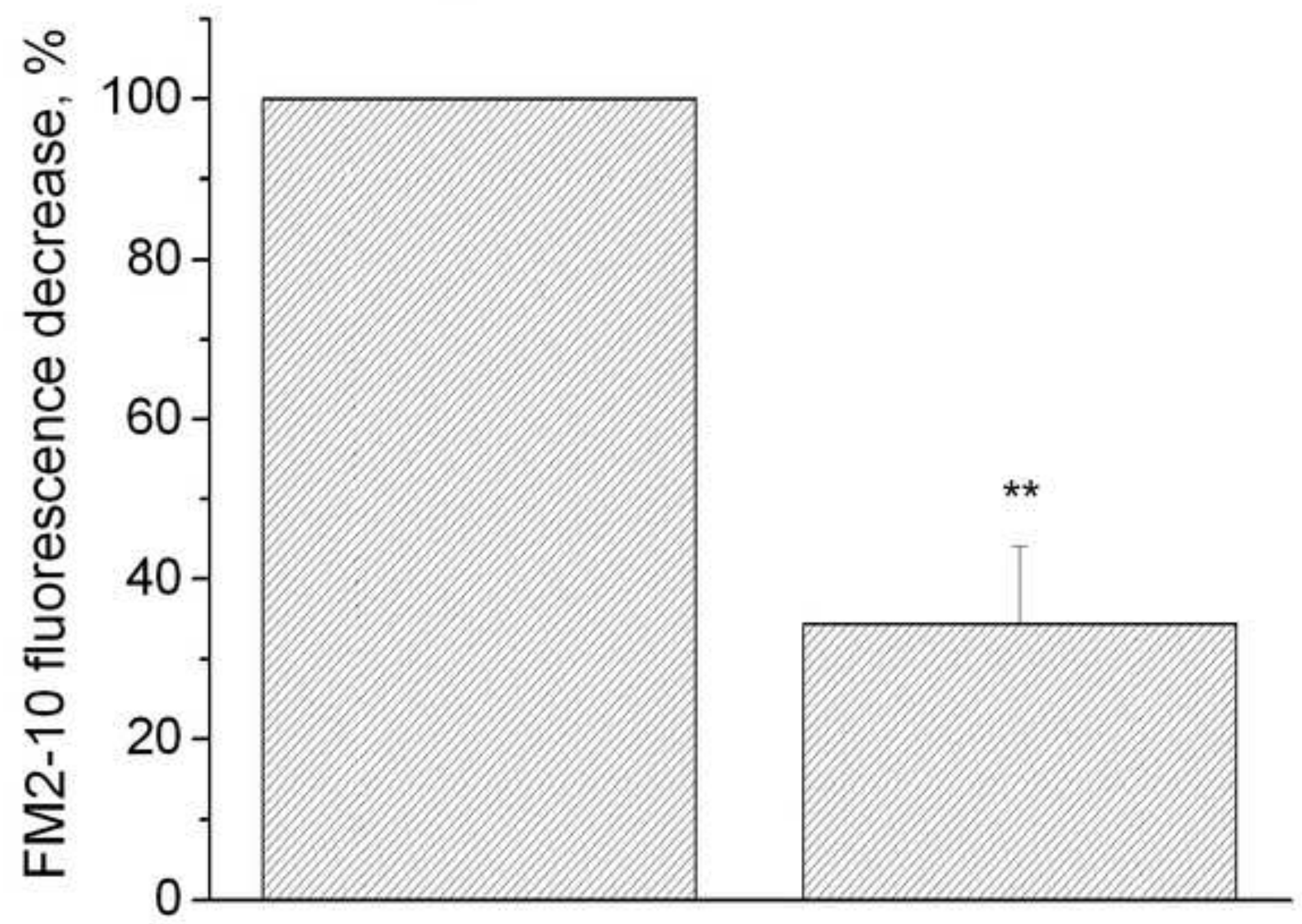


Con

BHB

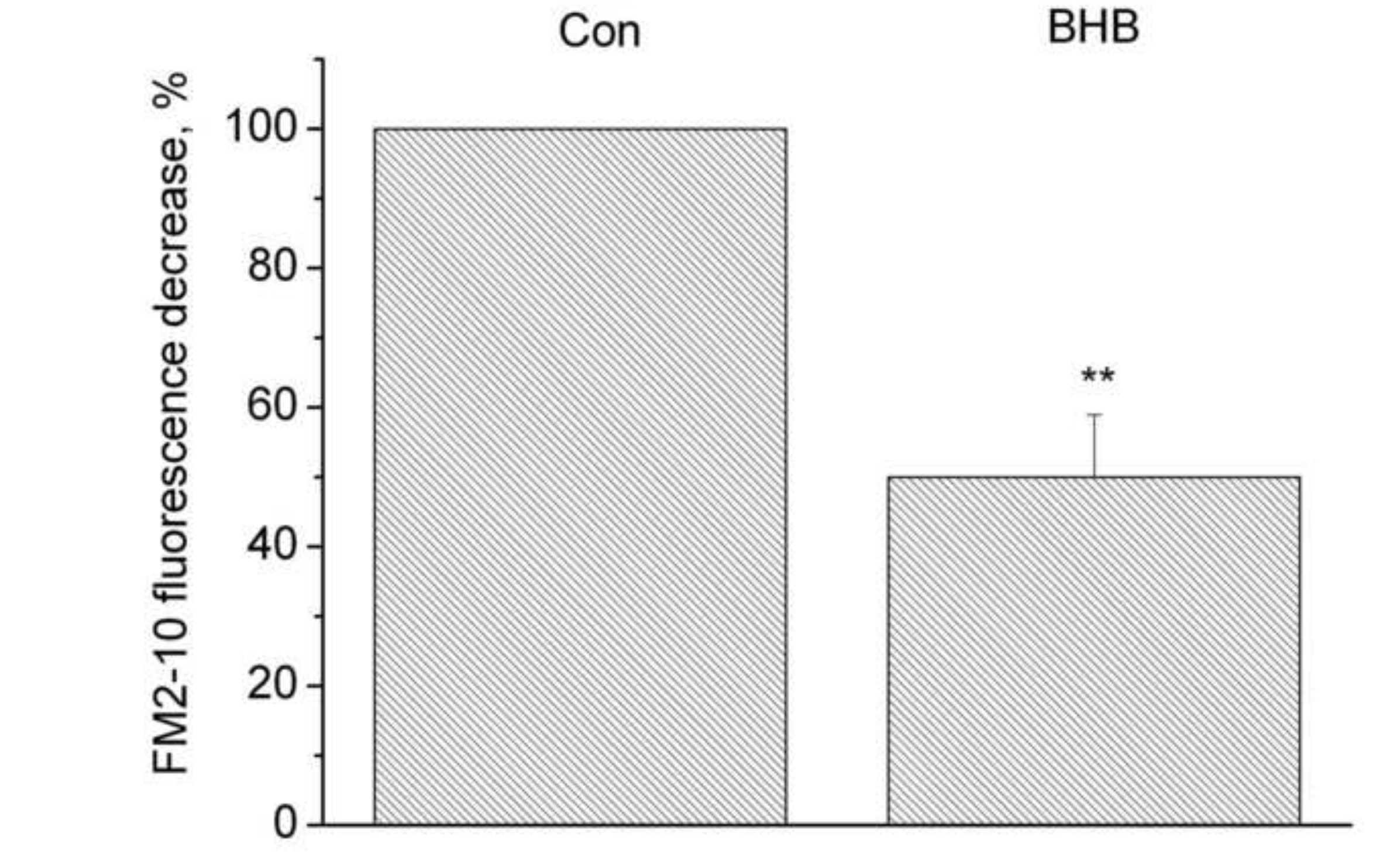

政
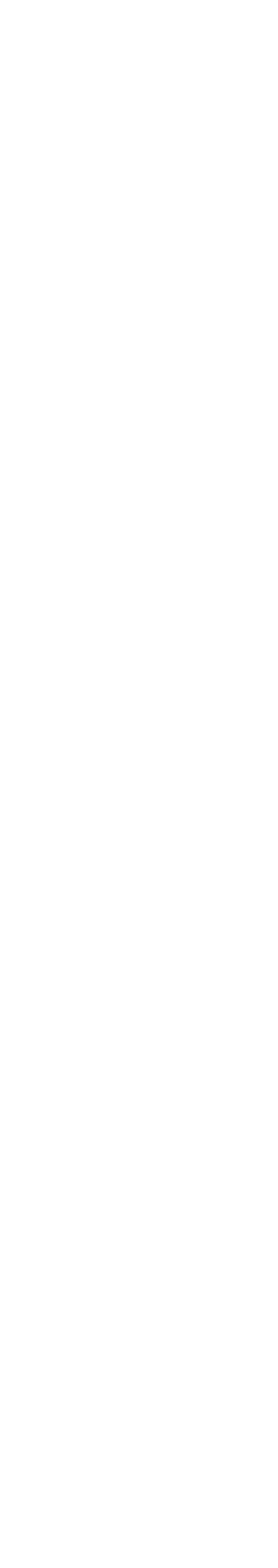


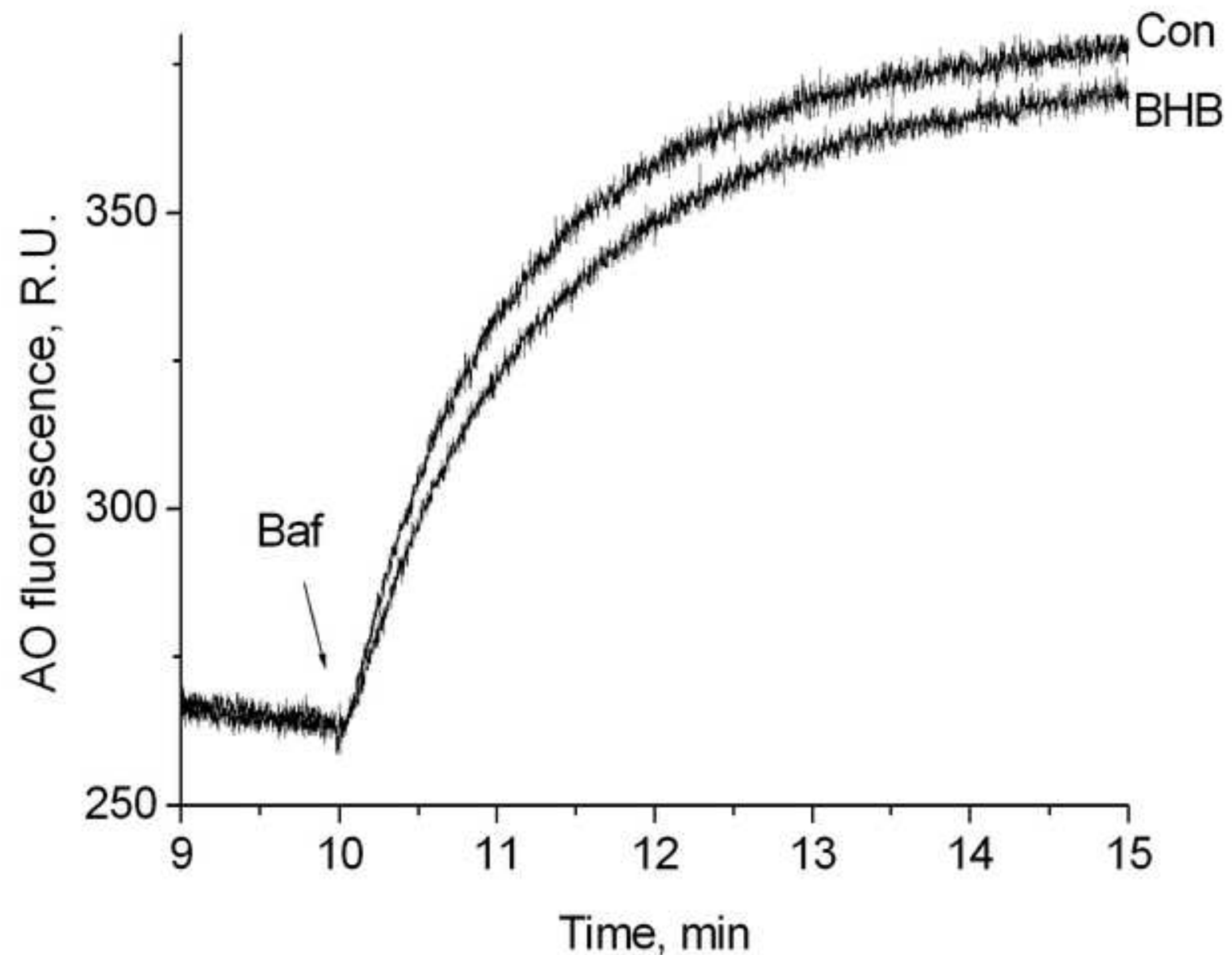


Con

\section{$\mathrm{BHB}$}

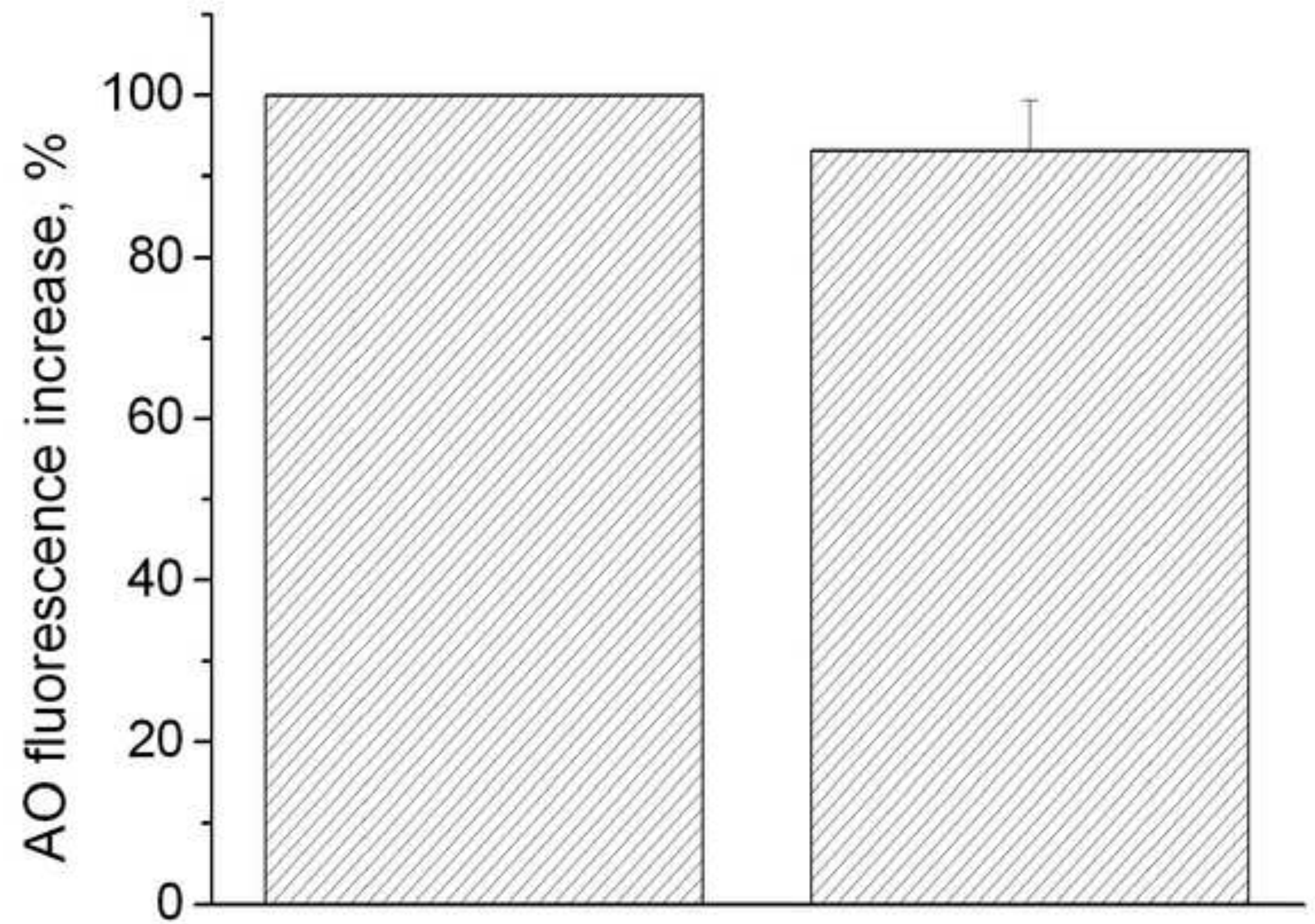




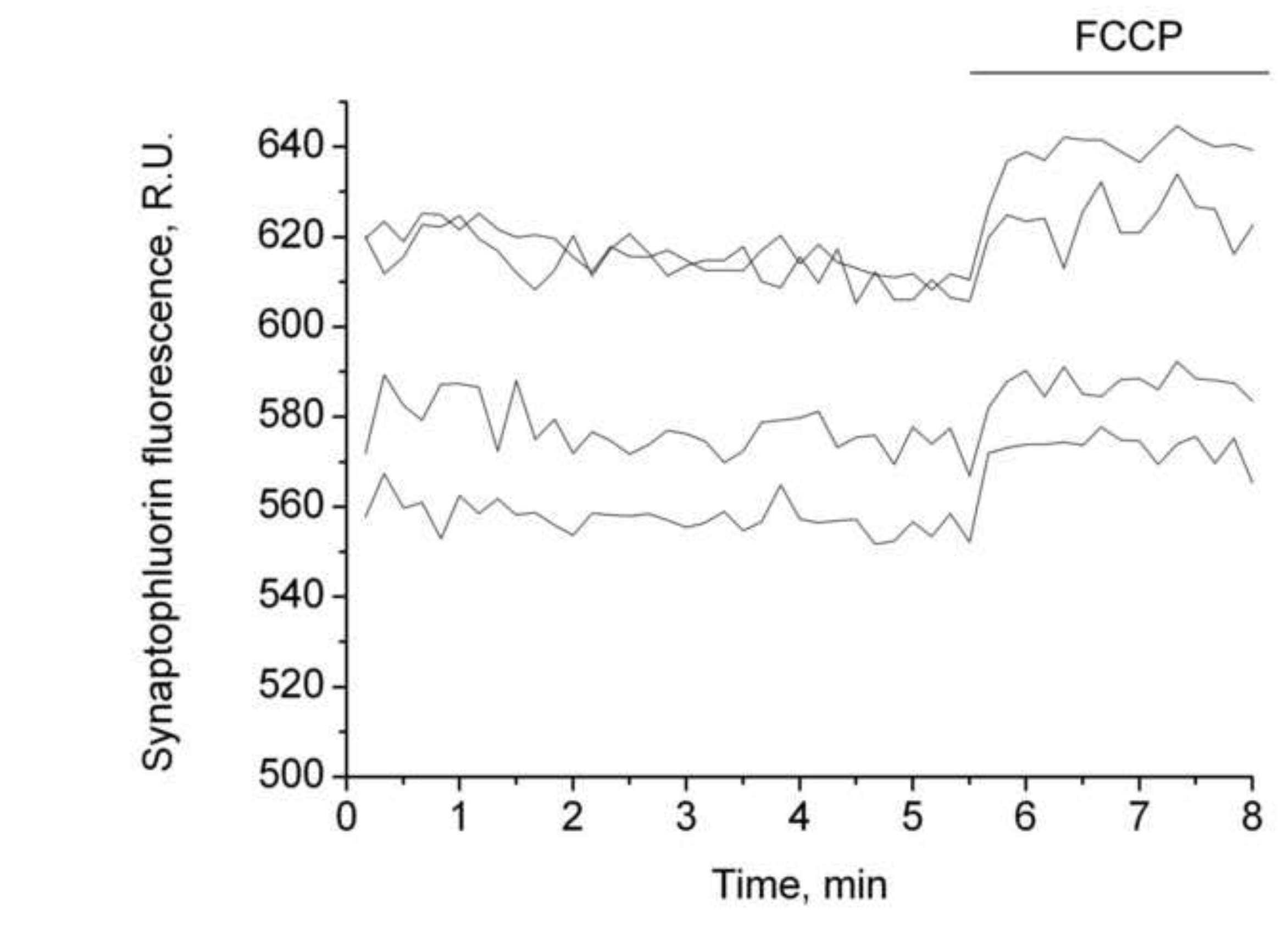

.

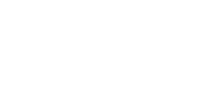

.

\section{FCCP}




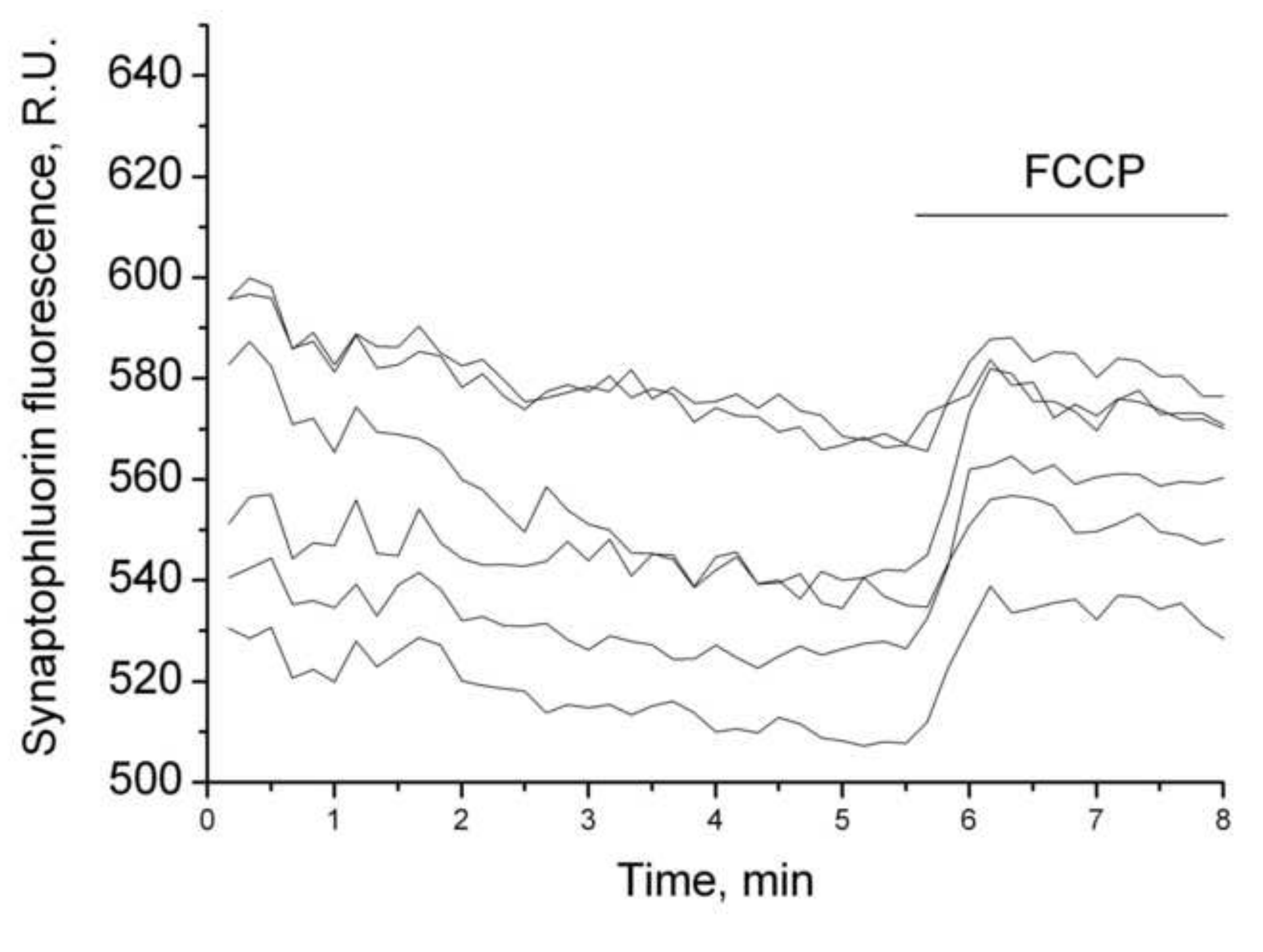

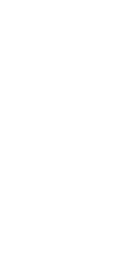

.

.

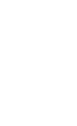


FCCP

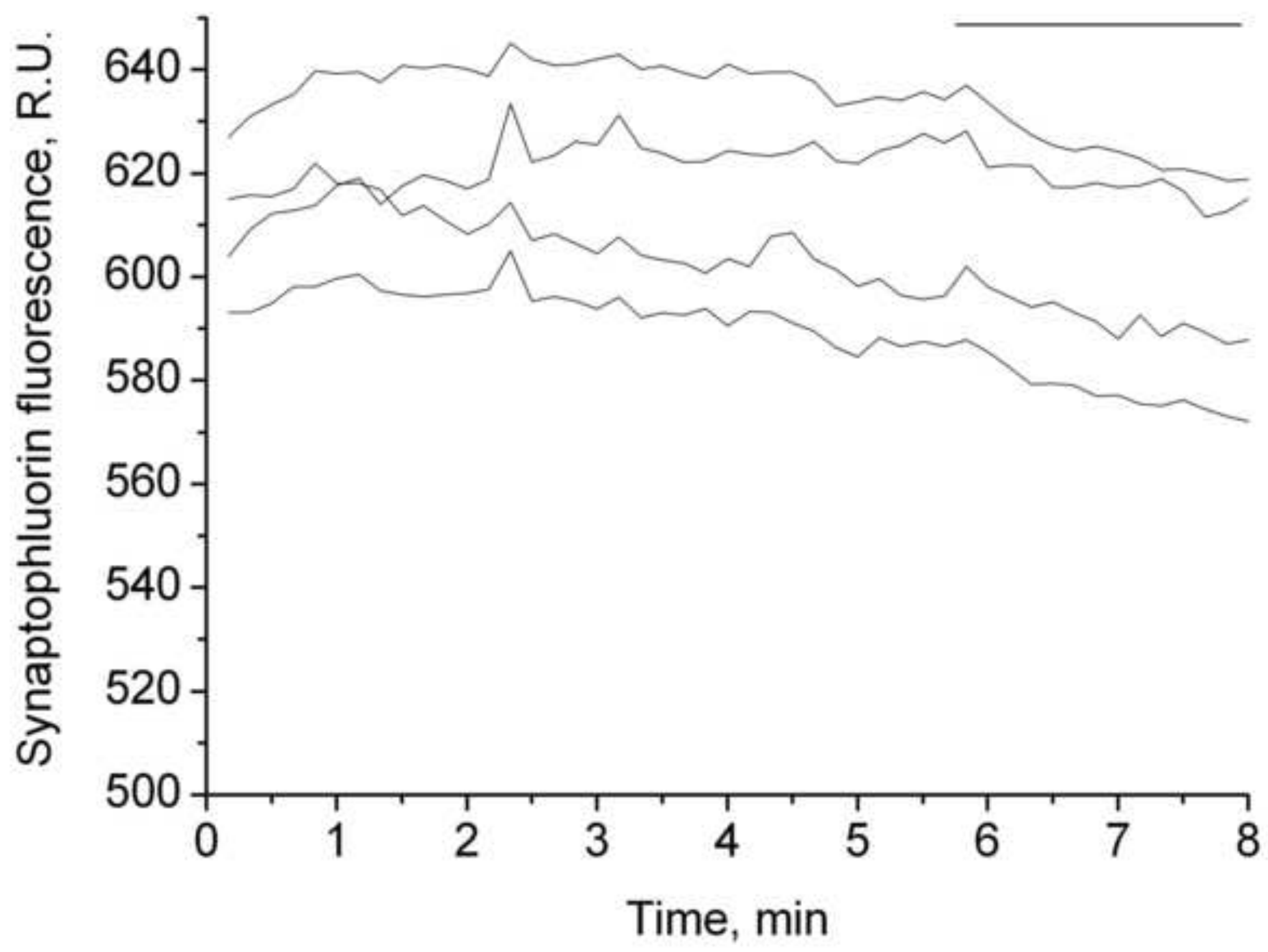




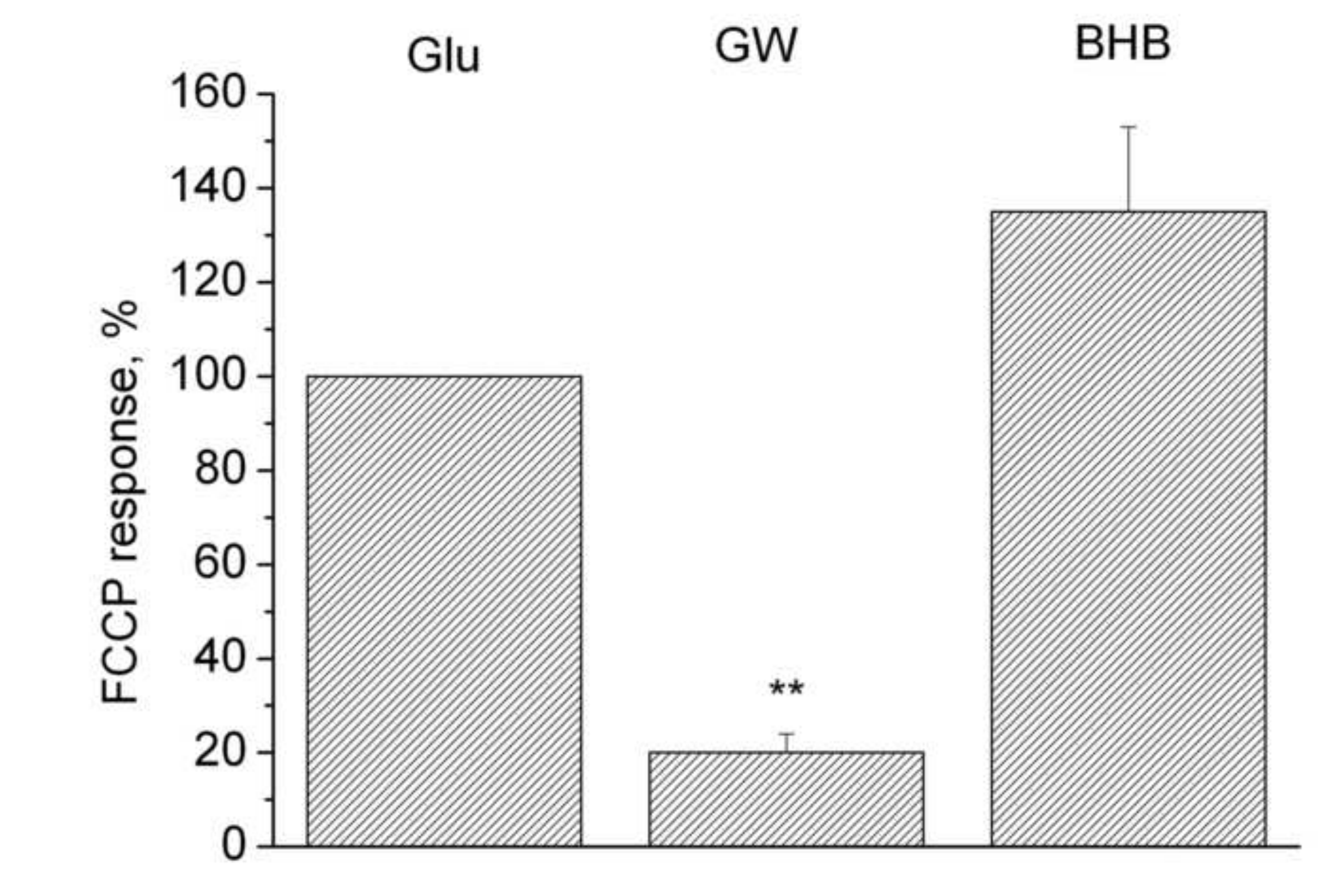

BHB

列

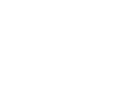

.




\begin{tabular}{|c|c|}
\hline Medium & Composition (in mM) \\
\hline A, synaptosomal, standard glucose-containing & $\begin{array}{l}132 \mathrm{NaCl}, 5 \mathrm{KCl}, 10 \text { glucose, } 1.3 \mathrm{MgCl}_{2}, 1.2 \\
\mathrm{NaH}_{2} \mathrm{PO}_{4}, 15 \mathrm{HEPES}, 5 \text { Tris, pH } 7.4\end{array}$ \\
\hline B, synaptosomal, ketone body containing & $\begin{array}{l}132 \mathrm{NaCl}, 5 \mathrm{KCl}, 8 \text { sodium DL- } \beta- \\
\text { hydroxybutirate, } 1.3 \mathrm{MgCl}_{2}, 1.2 \mathrm{NaH}_{2} \mathrm{PO}_{4}, 15 \\
\text { HEPES, } 5 \text { Tris, } \mathrm{pH} 7.4\end{array}$ \\
\hline C, synaptosomal, mixed & $\begin{array}{l}132 \mathrm{NaCl}, 5 \mathrm{KCl}, 4 \text { sodium DL- } \beta- \\
\text { hydroxybutirate, } 4 \text { glucose, } 1.3 \mathrm{MgCl}_{2}, 1.2 \\
\mathrm{NaH}_{2} \mathrm{PO}_{4}, 15 \mathrm{HEPES}, 5 \text { Tris, } \mathrm{pH} 7.4\end{array}$ \\
\hline D, synaptosomal, pyruvate containing & $\begin{array}{l}132 \mathrm{NaCl}, 5 \mathrm{KCl}, 4 \text { sodium pyruvate, } 1.3 \\
\mathrm{MgCl}_{2}, 1.2 \mathrm{NaH}_{2} \mathrm{PO}_{4}, 15 \text { HEPES, } 5 \text { Tris, } \mathrm{pH} \\
7.4\end{array}$ \\
\hline E, synaptosomal, energy substrate free & $\begin{array}{l}132 \mathrm{NaCl}, 5 \mathrm{KCl}, 1.3 \mathrm{MgCl}_{2}, 1.2 \mathrm{NaH}_{2} \mathrm{PO}_{4}, 15 \\
\text { HEPES, } 5 \text { Tris, pH } 7.4\end{array}$ \\
\hline $\begin{array}{l}\text { F, depolarizing synaptosomal, standard } \\
\text { glucose-containing }\end{array}$ & $\begin{array}{l}77 \mathrm{NaCl}, 60 \mathrm{KCl}, 10 \text { glucose, } 1.3 \mathrm{MgCl}_{2}, 1.2 \\
\mathrm{NaH}_{2} \mathrm{PO}_{4}, 15 \mathrm{HEPES}, 5 \text { Tris, pH } 7.4\end{array}$ \\
\hline $\begin{array}{l}\text { G, depolarizing synaptosomal, ketone body } \\
\text { containing }\end{array}$ & $\begin{array}{l}77 \mathrm{NaCl}, 60 \mathrm{KCl}, 8 \text { sodium DL- } \beta- \\
\text { hydroxybutirate, } 1.3 \mathrm{MgCl}_{2}, 1.2 \mathrm{NaH}_{2} \mathrm{PO}_{4}, 15 \\
\text { HEPES, } 5 \text { Tris, pH } 7.4\end{array}$ \\
\hline I, neuronal, standard glucose-containing & $\begin{array}{l}136 \mathrm{NaCl}, 2.5 \mathrm{KCl}, 10 \mathrm{HEPES}, 10 \text { glucose, } 2 \\
\mathrm{CaCl}_{2}, 1.3 \mathrm{MgCl}_{2}, \mathrm{pH} 7.4\end{array}$ \\
\hline $\mathrm{J}$, neuronal, ketone body-containing & $\begin{array}{l}136 \mathrm{NaCl}, 2.5 \mathrm{KCl}, 10 \mathrm{HEPES}, 8 \mathrm{DL}-\beta- \\
\text { hydroxybutiric acid, } 2 \mathrm{CaCl}_{2}, 1.3 \mathrm{MgCl}_{2}, \mathrm{pH} \\
7.4\end{array}$ \\
\hline $\mathrm{K}$, neuronal, energy substrate free & $\begin{array}{l}136 \mathrm{NaCl}_{2} 2.5 \mathrm{KCl}, 10 \mathrm{HEPES}, 2 \mathrm{CaCl}_{2}, 1.3 \\
\mathrm{MgCl}_{2}, \mathrm{pH} 7.4\end{array}$ \\
\hline
\end{tabular}

Table I. Composition of incubation media. 